\title{
Allan Sekula, du charbon à la mer : matérialisme culturel et réalisme critique
}

Allan Sekula, from the Coal to the Sea: Cultural Materialism and Critical Realism

Florent Le Demazel

\section{OpenEdition}

Journals

Édition électronique

URL : https://journals.openedition.org/itti/1472

DOI : $10.4000 /$ itti. 1472

Éditeur

Université de Poitiers

\section{Référence électronique}

Florent Le Demazel, «Allan Sekula, du charbon à la mer : matérialisme culturel et réalisme critique », Images du travail, travail des images [En ligne], 10 | 2021, mis en ligne le 20 février 2021, consulté le 26 janvier 2022. URL : http://journals.openedition.org/itti/1472 ; DOI : https://doi.org/10.4000/itti.1472

Ce document a été généré automatiquement le 26 janvier 2022.

Images du travail, travail des images 


\section{Allan Sekula, du charbon à la mer : matérialisme culturel et réalisme critique}

Allan Sekula, from the Coal to the Sea: Cultural Materialism and Critical Realism

Florent Le Demazel

1 Photographe, réalisateur, commissaire d'exposition, historien de la photographie et enseignant au California Institute of the Arts, Allan Sekula (1951-2013) est longtemps resté méconnu en France, même si la tendance a commencé à s'inverser au début de la décennie précédente. Plusieurs textes majeurs ont été traduits par Marie Muracciole, tandis que la Criée, le centre d'art contemporain de Rennes, l'a invité à présenter The Dockers' Museum, une exposition tirée de ses propres collections auxquelles Sekula a consacré les dernières années de sa vie ${ }^{1}$. Signalons également un colloque au Centre Pompidou, quelques semaines avant sa mort.

Dans cet article, nous nous proposerons d'abord de revenir sur la pensée théorique et historiographique de l'auteur, avant de nous arrêter sur un essai essentiel, proposant une relecture croisée de l'avènement du capitalisme industriel et des usages de l'image du travail ouvrier. Enfin, nous verrons comment l'artiste a, dès ses débuts, prolongé et parfois confronté ses idées à la pratique, la sienne propre ainsi que celle d'artistes amis. Ce parcours dans une œuvre dense et militante nous mènera des profondeurs des galeries minières aux grands ports d'un capitalisme maritime et mondialisé.

\section{Pour une histoire culturelle matérialiste}

\section{1. Une photographie critique à rebours du modernisme}

3 Allan Sekula intègre l'Université de San Diego au début des années soixante-dix, où il suit les cours de Louis Marin, Herbert Marcuse ou John Baldessari, et découvre l'œuvre de Walter Benjamin. C'est également à cette époque qu'il rencontre Martha Rosler, Fred Lonidier et Phel Steinmetz, avec qui il forme le Groupe de San Diego: un groupe 
informel de photographes critiques et engagés, nés dans l'après-guerre, qui prennent ouvertement position dans le champ politique, et dont Sekula rédige en 1976 le manifeste, « Défaire le modernisme »².

L'une des cibles privilégiées de ce pamphlet pour une photographie critique est le «modernisme », ce courant documentaire qui émerge à la fin des années soixante sous la férule de John Szarkowski. Directeur du département photographie du MoMa, ce dernier entendait redéfinir les bases de la photographie documentaire à travers l'exposition New Documents ${ }^{3}$ (1967), où étaient convoquées les œuvres de Diane Arbus, Lee Friedlander et Garry Winogrand. Dans son carton introductif, Szarkowski présentait ces auteurs ainsi : « Lors de la dernière décennie, une nouvelle génération de photographes a dirigé l'approche documentaire vers des fins plus personnelles. Leur but n'a pas été de réformer la vie, mais plutôt de la connaître. Leur travail trahit une sympathie - quasiment une affection - envers les imperfections et les fragilités de la société. » À la différence de la photo documentaire des années trente, marquée par les commandes de la Farm Security Administration (FSA), la vision moderniste évacue toute trace du monde social pour se replier sur l'immédiateté sensible de la photographie, considérée comme une activité autonome. La subjectivité de l'auteur devient seul critère de validation artistique, et la signification de l'image doit se chercher dans la photo elle-même, avec éventuellement le recours à quelques éléments de compréhension biographiques. Ce courant préconise un mode d'appréhension contemplative de l'image, calqué sur la peinture ${ }^{4}$ : il fallait affirmer, selon le mot de Peter Galassi, que « la photographie n'était pas un bâtard abandonné par la science sur le perron de l'art, mais un enfant légitime de la tradition picturale occidentale " (Galassi, 1981, 12). Cette " réclusion dans le formalisme » (Sekula, 1976-78), appuyée par l'autorité légitimatrice du musée, encourage la spéculation autour du marché de l'art photographique, qui connaît alors un véritable essor à travers l'expansion des galeries.

5 Sekula prend donc le contre-pied de cette vision moderniste pour adopter une approche résolument matérialiste de la photographie, tant dans ses conditions de conception et de fabrication que dans sa réception et ses effets de pouvoir et de domination. Ce qui l'intéresse, résume-t-il, c'est de comprendre « la production sociale, la circulation et la réception des photographies dans une société fondée sur la production et l'échange de marchandises »(Sekula, 1984, 58). La photographie est suspectée de participer au fétichisme de la marchandise en faisant disparaitre les rapports de production et en humanisant les différents aspects du travail industriel moderne: le médium a contribué à recouvrir d'un voile «sentimentalement acceptable » une « esthétique de l'exploitation » (Sekula, 1983).

Détricoter ce voile exige de prendre les photographies non comme des objets d'art privilégiés, uniques et autonomes, mais comme des artefacts culturels quotidiens, opérant à tous les niveaux de notre culture. De fait, l'historien s'attache à penser le caractère social du médium, à travers des sources la plupart du temps délaissées par les institutions dominantes. Il s'intéresse moins à des auteurs consacrés qu'à des lieux de pouvoir - la police, le musée, les sciences du travail - ou à des publications de la culture de masse : les magazines à grand tirage (Life, Fortune...), la presse régionale ou industrielle (rapports d'entreprise, journaux syndicaux, catalogues de vente par correspondance, etc.). Ces sources seront la base d'une «histoire culturelle matérialiste» (Sekula, 1983), une histoire de l'usage courant de la pratique 
photographique, qui cherche à analyser la photographie au sein d'un vaste réseau de rapports sociaux, à travers lequel elle façonne une " économie de l'imaginaire » :

«Comment la photographie a-t-elle servi à légitimer et normaliser les relations de pouvoir existantes? Comment s'est-elle fait la voix de l'autorité? Quelles résistances encourage-t-elle et renforce-t-elle? Comment la mémoire historique et sociale est-elle préservée, transformée, restreinte ou obturée par les photographies? Quel avenir promettent-elles, quel avenir oublient-elles? Au sens le plus large, ces questions concernent les manières dont la photographie construit une économie de l'imaginaire.» (Sekula, 1983, l'auteur souligne)

\section{2. Un matérialisme historique et sémantique}

7 Se réclamant d'un double héritage de Karl Marx et de Marcel Duchamp, Sekula pense donc la photographie comme prise dans un entrelacs de structures - sociales, économiques, technologiques et culturelles - qui la détermine, de sa fabrication à sa réception. Il lui fallait donc sortir de l'illusion d'une discipline autonome - l'histoire de l'art -, ce qui se conçoit d'emblée par les bibliographies mobilisées. Dans ses essais, Sekula replace les images dans une généalogie, dans une tradition représentative qui puise tant dans l'histoire des techniques (Lewis Mumford), l'histoire économique (Karl Marx, Friedrich Engels, Harry Braverman), dans la littérature (George Orwell, Herman Melville), dans la sémiologie (Roland Barthes, Roman Jakobson, Ferdinand de Saussure), en plus des historiens de la photographie traditionnels. Ces textes foisonnants servent à poser les fondations d'une histoire globale, indispensable à la compréhension d'ensembles plus petits: La Photographie, entre travail et capital (1983) prend la forme d'un "triptyque ", où la partie centrale consiste en une longue généalogie du réalisme technique, débouchant sur l'analyse d'un fonds archivistique d'un photographe mineur. Pour le livre et l'exposition Fish Story (1989-1995), Sekula rédige deux longs textes sur les représentations picturales de la mer, de la peinture marine du Siècle d'or hollandais aux photographies de marins de August Sander et Walker Evans, en passant par Moby Dick et l'arrivée en voilier d'Engels à Londres, au début de La Situation de la classe ouvrière en Angleterre en $1844^{5}$. Se dégagent les bases d'une "herméneutique " sekulienne, repérable jusque dans ses œuvres artistiques, où l'étude d'un sujet historique (le travail marin ou souterrain) est inséparable de l'analyse critique de la tradition picturale que ce sujet a générée, liant dans une même pensée les mouvements sociaux qui agitent l'infrastructure et leurs expressions superstructurelles. Une telle analyse révèle aussi bien souvent la constitution d'un savoir politique, où l'image joue un rôle dans la domination d'une classe sur une autre.

Loin d'être la "fille spirituelle de la peinture", la photographie se situe donc au croisement des structures qui façonnent le monde réel et son appréhension: s'appuyant sur Mikhail Bakhtine (1977), Sekula la voit comme une phase d'énonciation dans un échange social permanent et quotidien. Or, «tous les messages sont des manifestations d'intérêt. Et aucun modèle critique ne peut ignorer que les intérêts sont en lutte dans le monde réel» (Sekula, 1974, 67). Pour le théoricien, il n'y a pas d'« ontologie de l'image photographique » (André Bazin) ou de "ça-a-été » (Roland Barthes), concepts selon lesquels le médium, par son objectivité mécanique, nous obligerait à croire à l'existence de l'objet représenté. Pour notre auteur, les conditions matérielles présidant à la réalisation de l'image priment : on pourrait parler d'un «çaa-été-fait ». Il faut entendre par là une " ontologie » résolument immanente au monde social : « La seule vérité "objective" que les photographies puissent offrir est l'assertion 
que quelqu'un ou quelque chose [...] se trouvait quelque part et a capté une image. Tout le reste, tout ce qui dépasse la trace imprimée, est interprétation » (Sekula, 1976-78, 148, l'auteur souligne). Une image est toujours réalisée dans un cadre donné, pour une fonction précise, et prendra sens selon un contexte qui ne dépend pas d'elle.

Et précisément, la question de l'interprétation et du sens est un apport crucial de l'auteur à la théorie critique. Au lieu d'en faire le lieu d'une suspension kantienne du jugement, Sekula, influencé par le Barthes des années soixante, insiste au contraire sur le rôle du discours, du texte qui entoure l'image et qui en influence la lecture. Dans sa pensée, le sens que l'on donne à une photographie est fondamentalement contingent : "La photographie constitue un énoncé "incomplet", un message dont la lisibilité repose sur une matrice externe de conditions et de présupposés. C'est-à-dire que la signification d'un message photographique est nécessairement déterminée par un contexte» (Sekula, 1974, 67). C'est tout le paradoxe du médium, souligné par Muracciole: «la capacité à informer de ce dispositif de duplication du visible est entièrement tributaire d'un contexte qu'il ne rend pas visible » (Muracciole, 2013, 26).

Or, ces conflits d'intérêts et d'interprétations risquent de disparaître quand la photo glisse dans une zone de perte sémantique, comme l'archive, l'exposition ou l'édition. Et d'un contexte à l'autre, la "réalité » photographiée sera radicalement différente : la même photo n'appelle pas la même interprétation quand elle illustre un reportage ou quand elle est accrochée sur les murs d'un musée. Dès lors, pour faire œuvre d'historien, il est absolument nécessaire de retrouver le support social d'origine. En retrouvant cet échange initial apparaît la fonction première de la photo, qui a déterminé sa fabrication, et avec elle les dialogues et les relations de pouvoir qui se sont tissés autour des prises de vue.

\section{3. Sortir de l'équivalence : lire l'image par le bas}

11 La photographie se caractérise donc pour Sekula par son caractère social, et ce dernier se perd quand l'image est remisée et inventoriée dans un fonds: "les archives établissent une relation d'équivalence visuelle abstraite entre les images » (Sekula, 1983). C'est ce règne de l'équivalence que l'on retrouve dans l'exposition mondialement connue The Family of Man, sur laquelle Sekula n'a cessé de revenir. Son commissaire, Edward Steichen, entendait illustrer les similitudes humaines autour du globe par la seule comparaison photographique. De fait, en présentant la naissance, la famille, l'amour ou la mort comme des expériences vécues uniformément à travers le monde, l'exposition fait disparaitre purement et simplement les différences culturelles entre les peuples. Détachés de tout référent social ou historique et organisés autour d'un modèle occidental (famille nucléaire, citations bibliques, dégustation de Coca-Cola, mécène de la manifestation), les centaines de clichés pris sur les six continents allaient en réalité participer d'une forme précoce de soft-power, l'exposition ayant plusieurs fois voyagé dans des pays victimes de l'impérialisme occidental ${ }^{6}$.

12 Parmi les thèmes rendus ici à l'état de fait universel, le travail n'est pas le moins problématique. Décrit comme une activité "naturelle», les évolutions structurelles historiques dans l'organisation de la production et la répartition de ses fruits en deviennent imperceptibles. Pour illustrer cette uniformisation, Sekula s'appuie sur une double page du catalogue, où deux photographies sont mises en vis-à-vis (figure 1$)^{7}$ : à gauche, Gjon Mili a photographié les mains d'une jeune femme dont les tâches et 
déplacements sont réduits au minimum par le management scientifique, et se répètent inlassablement au rythme de cadences intensives. À droite, les mains d'une fermière de l'Iowa, réduite à la misère par la crise économique de 1929, photographiées en 1936 par Russell Lee. Les déterminismes historiques qui expliquent les situations respectives de ces travailleuses sont éclipsés par le parallèle visuel. Plus encore, une citation du Deutéronome, en bas de la page de droite, semble délivrer la clé de la comparaison: "Béni soit tout travail que tu entreprendras de tes mains. » Une telle lecture dénie aux ouvrières le caractère à la fois violent et historiquement compréhensible de leurs dominations respectives, transformées en une illustration positive du commandement divin.

Figure 1 : Steichen E. (1955), The Family of Man, Museum of Modern Art, New York, p. 78-79.

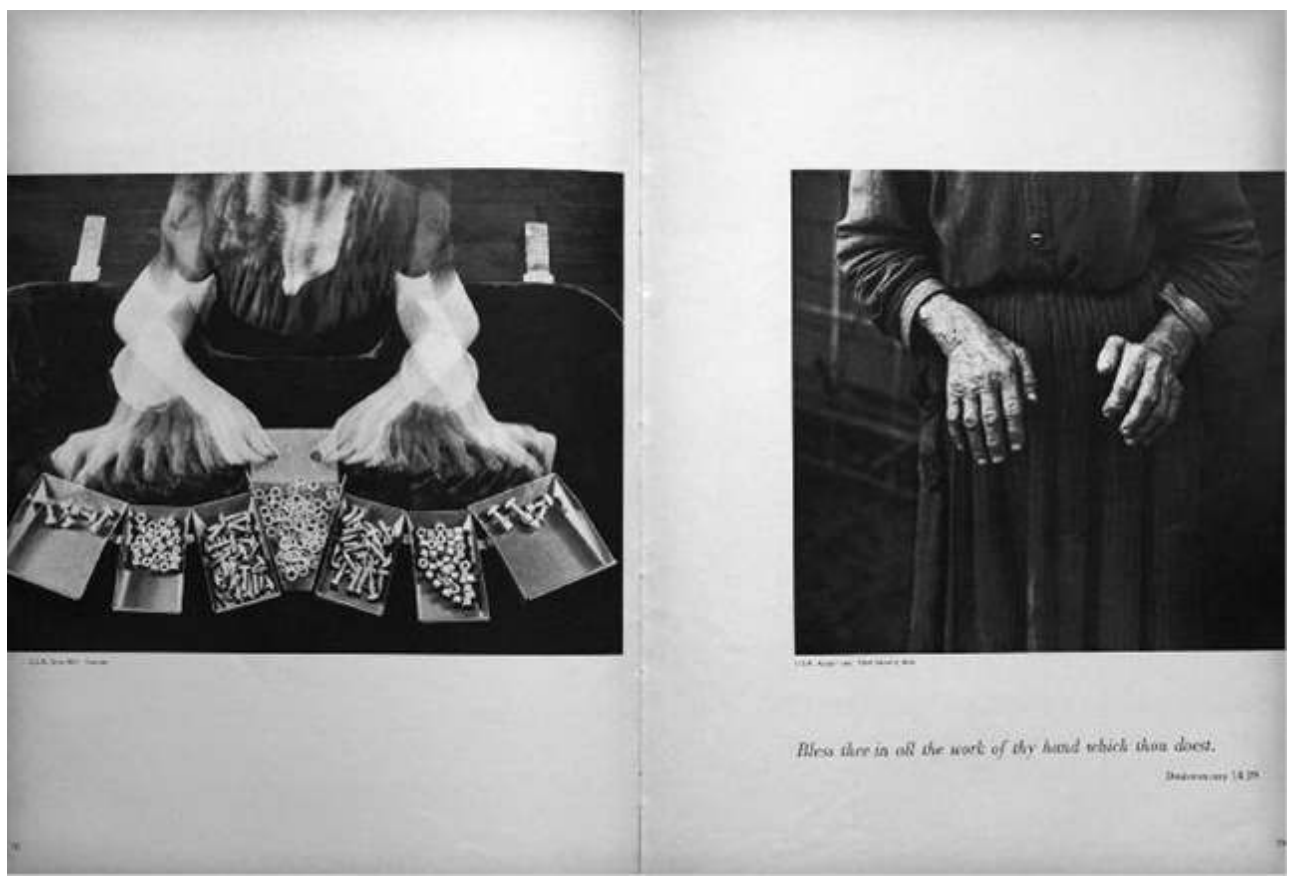

L'exposition illustre ainsi la thèse de Benjamin que Sekula fait sienne selon laquelle: "Il n'est aucun document de culture qui ne soit aussi un document de barbarie.» Contre cette barbarie de la culture bourgeoise, la tâche de l'historien matérialiste était, selon Benjamin de «brosser l'histoire à rebrousse-poil " (Benjamin, 1940). Pour Sekula, écrire l'histoire à rebrousse-poil ou regarder la photographie contre le courant, cela peut vouloir dire sortir de la fascination esthétique pour observer «par le bas, depuis une position solidaire de ceux qui sont déplacés, déformés, réduits au silence ou rendus invisibles par les machines du profit et du progrès " (Sekula, 1983). Cette position solidaire exige une empathie critique : une empathie soucieuse de donner un visage et une histoire à ceux qui sont représentés, sans renoncer à comprendre leur situation sociale, pour se donner les moyens de la changer. Cette lecture "par le bas » est au cœur de la rédaction du premier grand essai historique de Sekula, dans lequel l'auteur relit les évolutions de la photographie à partir des perfectionnements d'un réalisme pictural, intimement lié aux perfectionnements de la grande industrie et à la place croissante prise par la profession d'ingénieur. 


\section{Entre travail et capital : une généalogie du réalisme technique}

\section{1. Une exploration minutieuse des mouvements et des vies prolétaires}

En 1983, suite à la découverte des archives de Leslie Shedden ${ }^{9}$, artisan photographe du bassin houiller de Cap-Breton (Canada), Sekula est invité à rédiger une histoire des représentations du travail souterrain : La Photographie, entre travail et capital. L'essai est une application concrète de son matérialisme culturel, avec en ligne de mire cette question: "quel rôle les images ont joué dans le développement historique du capitalisme? » L'auteur y observe "l'émergence d'un langage visuel du capitalisme ", lui-même consécutif à l'ascension d'une classe sociale, la bourgeoisie, désireuse d'accaparer les savoir-faire des artisans, organisés en corporations, puis en syndicats. L'image va servir de point d'appui pour permettre à cette bourgeoisie nouvelle de connaître, comprendre puis « superviser » le travail manuel.

Cette trajectoire historique débuterait avec le De Re Metallica (1556) de Georgius Agricola (1494-1555). Pour ce premier traité de gestion des affaires minières, Agricola a fait réaliser de nombreuses gravures sur bois afin de suppléer à l'imprécision des mots. Le but est de former des gestionnaires: le recueil doit donc forger « un logos, un discours raisonné sur un ensemble technique à but d'administration » (Garçon, 2010). Il faut connaitre l'industrie et son lexique pour commander aux artisans. Le De Re Metallica fixe ainsi les bases d'un modèle de savoir centralisé : le savoir de l'ingénieur, destiné à optimiser scientifiquement l'investissement financier. C'est ce même modèle que reprennent Denis Diderot et Jean D'Alembert, qui envoient également des dessinateurs dans les ateliers afin de faire comprendre « en un coup d'œil » ce que les mots ne peuvent dire. Cependant, dans l'Encyclopédie, ce geste s'accompagne d'une dépréciation des savoirs empiriques au profit de la science et de la Raison. Les artisans sont suspectés de ne rien connaître à leurs machines, voire de mentir délibérément pour protéger leurs secrets professionnels ${ }^{10}$. Les planches montrent des scènes de travail abstraites et géométriques, où toute expérience sensible et sociale a disparu (figure 2). L'image occupe une fonction instrumentale dans un «circuit virtuel du savoir » (Sekula, 1983) : il faut mettre à disposition du savant un savoir empirique, afin que celui-ci l'observe et l'améliore, et s'en serve pour commander aux artisans, supposément dénués de raison. En ce sens, le Dictionnaire des Lumières constitue le « manuel conceptuel » du mode de production capitaliste : une division du travail, au sens marxiste du terme, entre ceux qui font - tâche de fabrication - et ceux qui savent - pouvoir de conception. 
Figure 2 : Diderot D. (1768), Recueil de Planches sur les Sciences, les Arts liberaux et les Arts mechaniques, Vle Volume, « Minéralogie, Ardoiserie de la Meuse », pl. IV. D'après Robert Bénart.

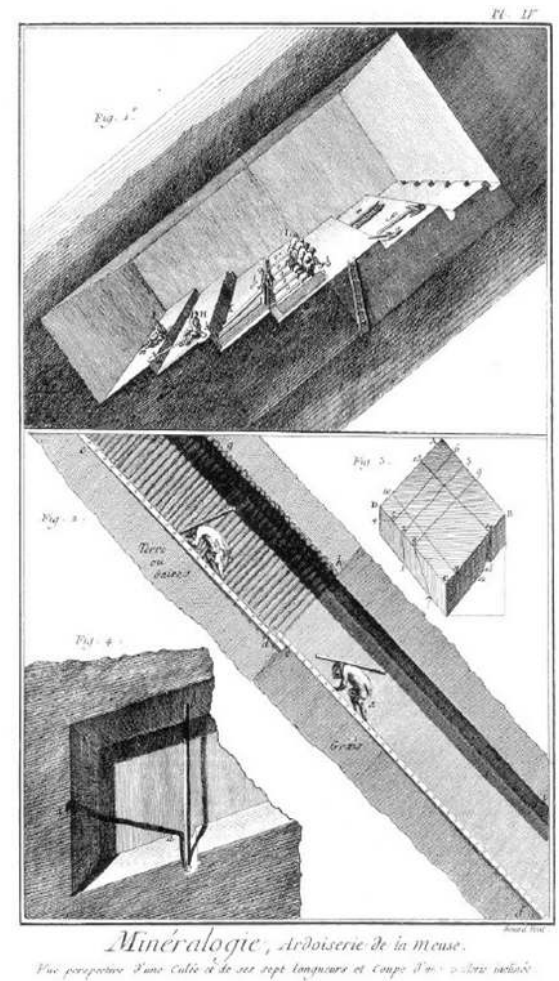

C'est ce même paradigme que l'on retrouvera chez Frederick Winslow Taylor. Simone Weil a bien montré que la grande victoire du père du management scientifique est d'avoir obtenu l'autorisation d'installer son laboratoire au sein de l'usine ${ }^{11}$, afin de pouvoir observer les ouvriers au travail et accaparer leur savoir. Comme le rappelle Weil, le souci de Taylor n'a jamais été de faire travailler mieux, mais de faire travailler plus. Pour cela, il était nécessaire de connaître les durées indispensables à telle ou telle opération: "Son but était d'ôter aux travailleurs la possibilité de déterminer euxmêmes les procédés et le rythme de leur travail et de remettre entre les mains de la direction le choix des mouvements à exécuter au cours de la production " (Weil, 1951, 301). Sekula relève en sus que dans son Management de l'atelier (1903), Taylor appuie sa division du travail sur une " archive centralisée » à l'intérieur de l'usine : "Le bureau d'information devrait inclure des catalogues de dessins (...) ainsi que tous les enregistrements et rapports de l'établissement tout entier. L'art d'indexer proprement l'information n'est en aucune manière un art simple, et autant que faire se peut, il devrait être contrôlé par un seul homme.» (Taylor, 1903, cité par Sekula, 1983). Le manager et l'ingénieur étaient les seuls détenteurs d'un savoir industriel, autrefois détenus par les ouvriers, et qui pouvaient être transmis sous forme d'images visuelles ${ }^{12}$. La photographie va transformer et accélérer le projet encyclopédique : trace physique de l'objet, elle en conserve aussi les mesures mathématiques. Dans son rapport enthousiaste à la Chambre en 1839, François Arago y voit un instrument de conquête colonial, un moyen d'accumuler des données et d'archiver le monde ${ }^{13}$. Elle servira également à rendre compte de l'évolution des chantiers lointains (dans les expositions internationales ou dans les albums dédiés) ou, aux États-Unis, à mobiliser la nation autour de la conquête de l'Ouest. Au mitan du XIX ${ }^{e}$ siècle, Britanniques et Américains y voient quant à eux un outil de moralisation des classes laborieuses. Pour Marc Aurelius 
Root, la reproductibilité technique du médium recèle un potentiel éveil culturel, et les portraits de famille pourraient resserrer les liens familiaux, renforçant chez l'ouvrier une "affection primale pour son foyer", dans un souci de cohésion sociale: "une nation n'est vertueuse et unie qu'à condition que les foyers qui la composent soient bien ordonnés et liés ensemble par une considération mutuelle. » (Root, 1864, 413).

Finalement, ces deux tendances - analyse des procès du travail, moralisation de la vie domestique - trouvent leur aboutissement dans l'expérimentation des disciples de Taylor, les époux Frank et Lilian Gilbreth. Ces derniers vont mettre au point un "chronocyclographe ", dérivé de la chronophotographie inventée par Étienne-Jules Marey : des ampoules sont fixées sur les membres actifs de l'ouvrier, et l'appareil de captation est doté d'un chronomètre. Le dispositif permet de synthétiser les gestes en un diagramme lumineux, afin de révéler un mouvement optimal (figure 3). Le mouvement efficient est montré en exemple aux autres ouvriers, et le sujet peut emporter l'image chez lui, afin d'être félicité par sa famille. Le management scientifique des Gilbreth est un "système total, s'étendant au-delà de l'usine pour englober la vie et le quotidien tout entier » (Sekula, 1983) : l'aliénation de l'ouvrier est récompensée et censée lui apporter de la joie.

Cette première trajectoire illustre donc une pénétration toujours plus profonde dans le geste et la vie de l'ouvrier, la mécanisation du réalisme pictural accompagnant l'automatisation des procès de production. L'image invite à lire et à connaître le corps du travailleur comme une ressource passive devant être optimisée par des savants courant après l'efficience productiviste et la paix sociale.

Figure 3 : Gilbreth F. et L. (1919), Applied Motion Study, Sturgis and Walton, Londres

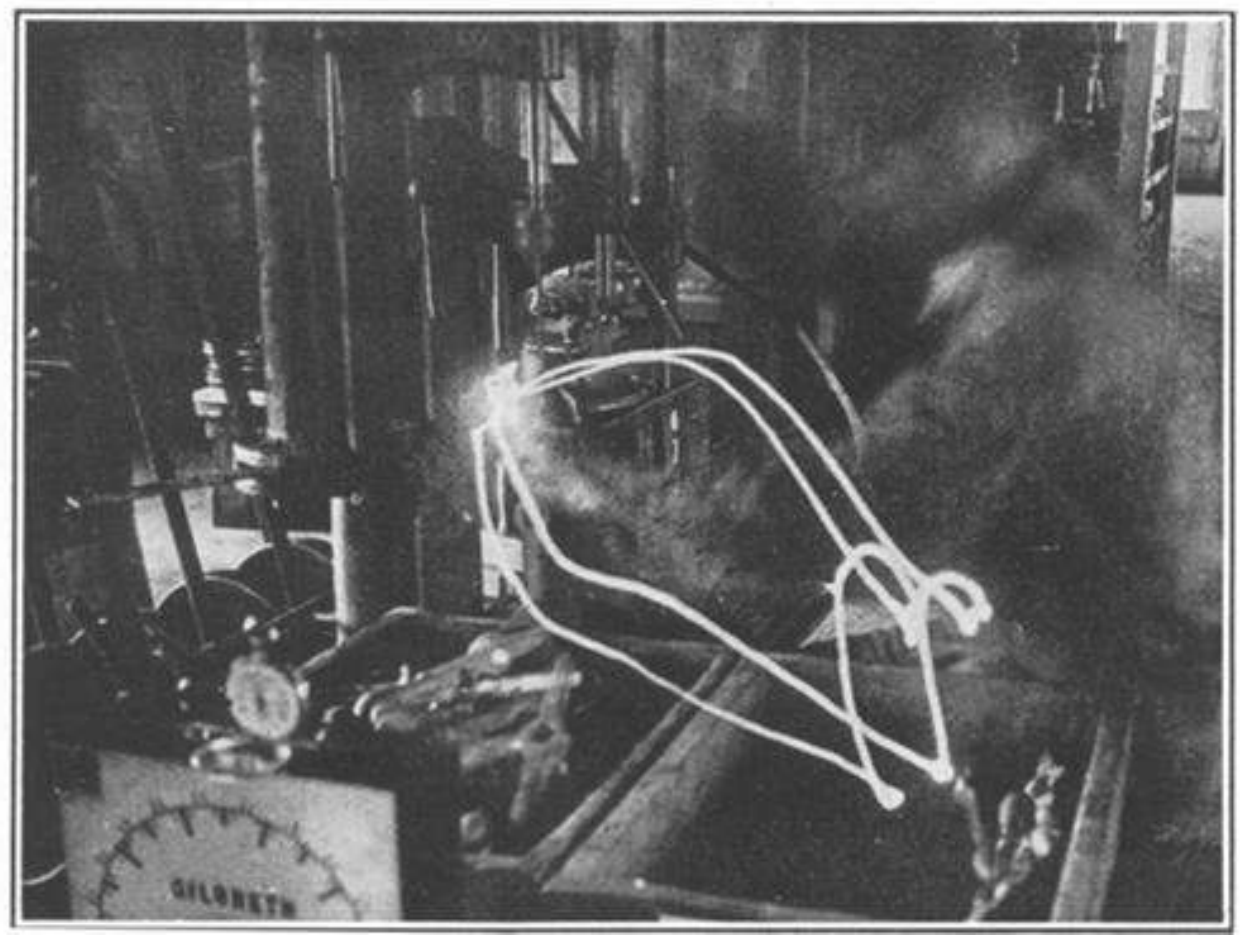




\section{2. La photographie et ses mondes imaginaires}

20 L'univers idéalisé des planches encyclopédiques a aussi eu une autre fonction, idéologique : il s'agissait de faire entrevoir au lecteur éloigné des arts mécaniques les potentialités d'un monde gouverné par la Raison. Là encore, la photographie va permettre à un tel projet de gagner en ampleur et en diffusion. Sekula prend pour exemple l'excursion de Nadar dans les catacombes de Paris en 1861, dans le but d'y réaliser les premières photographies souterraines (figure 4). Pour pallier le temps de pose de dix-huit minutes, dans une quasi-obscurité, le photographe met en scène des mannequins grimés en ouvriers. Sa série décompose un processus de travail, renvoyant l'image d'un monde souterrain occupé ${ }^{14}$, arraché aux ténèbres et transformé en espace de travail rationnel. Pour cela, Nadar a pu compter sur une alliée de poids : la lumière électrique, dont il vient de déposer une demande de brevet. Au même moment, celle-ci se généralise dans les usines, permettant l'implantation à grande échelle du travail de nuit, ainsi qu'un contrôle accru de l'ouvrier, en faisant disparaitre les coins de pénombre qui étaient autant de petits espaces de liberté : «[L'éclairage électrique] rend plus transparent, autrement dit il rationalise l'espace de l'usine ou de l'entrepôt " (Woronoff, 378) ${ }^{15}$.

21 Cette série de Nadar résonne avec les archives de Shedden. Son plus gros client était la Dominion Steel Company (Dosco). Productrice de machines d'exploitation, l'entreprise attendait du photographe qu'il mette en avant la puissance de la mécanisation. Les mineurs sont donc absents, ou ne sont au mieux que des appendices de la machine dans les clichés publiés dans les rapports annuels destinés aux actionnaires. On y chercherait en vain une expérience réelle de la mine. Bien plutôt, ces images se conforment au paradigme encyclopédique : des tableaux propres et bien éclairés, avec une perspective artificielle construite par un habile usage de l'éclairage électrique. Comme les clichés de Nadar, les archives Shedden paraissent terriblement figées : tantôt le travail est mis en pause et joué pour la photo ; tantôt les machines décrites en mouvement par la légende sont en réalité immobiles. La mécanisation elle-même est présentée comme une innovation bénéfique pour l'ouvrier puisque, loin de supprimer son emploi et de le mettre au chômage, elle lui procurera "une retraite bien méritée ». Ce qui fait dire à Sekula : «Même la plus fade déclaration en mots et en images de la part du service des relations publiques d'une entreprise contient un sous-texte marqué par les menaces et la peur (après tout, dans un régime capitaliste, chacun risque son poste) » (Sekula, 1983). 
Figure 4 : Nadar, Catacombes de Paris. Vue intérieure d'une galerie : déplacement des ossements, 1861. Épreuve à l'albumine sur papier.



Ce que révèlent ces archives, c'est qu'avec le développement industriel du capitalisme, la pratique photographique s'institutionnalise sous l'effet d'une double demande de documentation technique : une demande interne, essentiellement fonctionnelle, de la part des ingénieurs et des actionnaires; une demande externe, plutôt idéologique, voulue par la presse à grand tirage. Or, Sekula observe un renversement inattendu. Le début $\mathrm{du} \mathrm{XX}^{\mathrm{e}}$ siècle est traversé par l'opposition entre les ingénieurs, porteurs d'une vision «techniciste» et les réformateurs sociaux (travailleurs sociaux, avocats et sociologues sociaux-démocrates) dont Lewis Hine incarne le mieux le regard «humaniste ». Les photographies de ce dernier sont publiées dans des journaux, sur des affiches ou des tracts, afin de mobiliser l'opinion publique autour de l'évolution de la législation, que ce soit sur les conditions de travail ou le travail des enfants. Cette opposition techniciste / humaniste sera toutefois de courte durée, puisque l'humanisme photographique se fera "de plus en plus diffus et de moins en moins réformateur » (Sekula, 1983). Ainsi, en 1931, le magazine Fortune commande à Margaret Bourke-White un reportage sur les mineurs (Fig.5), agrémenté d'un commentaire positif sur la mécanisation: 
Figure 5 : Bourke-White (1931), « Hard Coal », Fortune, Février 1931.

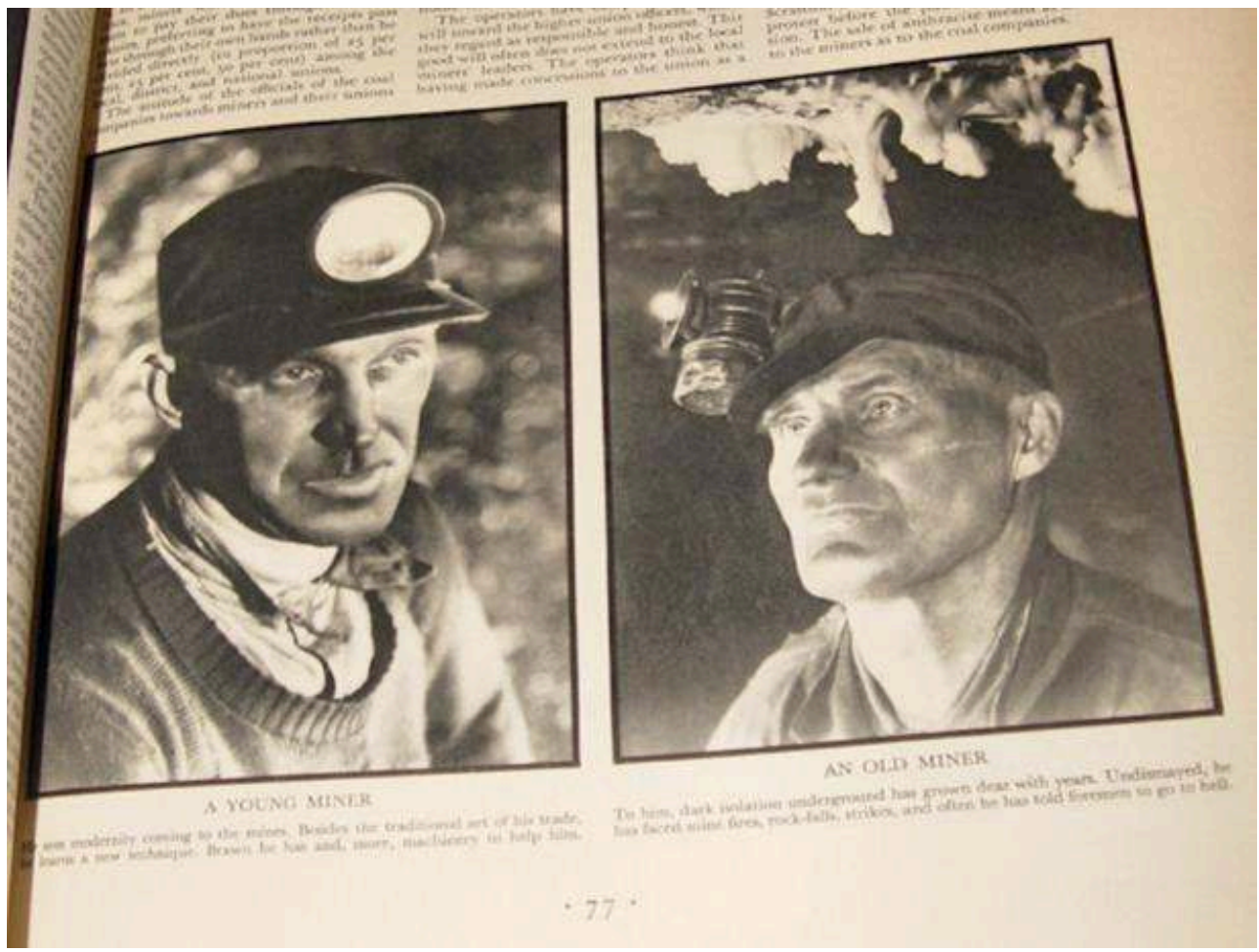

« UN JEUNE MINEUR. II voit venir la modernité dans les mines. En plus du métier traditionnel, il apprend de nouvelles techniques. Si fort soit-il, la machine l'aidera toujours. »

À un moment où l'organisation scientifique du travail gagne les galeries souterraines, privant le mineur de toute autonomie, la photo construit la fiction d'un ouvrier maître de ses gestes et de son destin, permettant ainsi au capitalisme "d'humaniser son image ». À la même époque, en France, le photographe François Kollar est appelé par les éditions Horizons de France à photographier des centaines d'entreprises françaises, dont les usines parisiennes de Renault et Citroën ${ }^{16}$. À cette occasion, il réalise le portrait en pleine page d'un ouvrier occupé à l'«ébarbage des cylindres au pistolet pneumatique ». L'homme, cadré aux cuisses, occupe toute l'image et semble détaché du reste de l'usine. Dans la partie basse de l'image, la pièce sur laquelle il s'affaire est elle aussi individualisée, donnant l'impression d'un ouvrier responsable de l'ensemble de son ouvrage. À l'avant-plan, un marteau et un burin bordent le cadre, renforçant l'apparence artisanale du travail. Pourtant, en y regardant de plus près, on s'aperçoit que le bloc ébarbé est posé sur un tapis roulant, et entouré de deux blocs identiques. Et puis, au second plan, profitant de l'arrêt momentané de la chaine nécessaire à la pose, un ouvrier s'active à son poste. Alors que le sujet mis en scène est on ne peut plus net, celui qui, derrière, travaille vraiment reste une silhouette floue. Un tel exemple est récurrent dans l'œuvre de Kollar, qui photographie très peu la chaine dans son ensemble et dans son urgence, préférant ponctionner sur elle des îlots de travail individuel où le temps parait comme arrêté, et le travailleur maître de ses mains. Dans une telle manière, les effets aliénants de la division du travail disparaissent au profit d'une autonomie et d'un savoir-faire de façade. C'est l'autre face de ce «langage visuel » du capitalisme, qui a tout à la fois servi à approfondir la division du travail et à en masquer les douloureuses conséquences. 
Ayant analysé le fonctionnement de cette rhétorique du réalisme industriel, Sekula va chercher à la subvertir par l'élaboration d'un "réalisme critique »: un mode de représentation conscient, d'une part, des forces et des lacunes du médium photographique, et d'autre part, de l'illusoire unité de l'existence du travailleur en régime capitaliste. C'est pourquoi Sekula artiste optera pour une esthétique fragmentaire: fragmentation des tâches et des corps par le travail automatisé; fragmentation des modes de témoignage, entre image, parole et commentaire; fragmentation des espaces de transit des marchandises, tentant de réassembler le gigantesque puzzle du commerce mondial.

Figure 6 : François Kollar (1932), «Ébarbage des cylindres au pistolet automatique », " Ch. L'Automobile, La Route... ", La France travaille, Horizons de France, Paris, T. II, p. 332.



\section{Pour un « réalisme critique » : un documentaire à contre-courant}

\section{1. Une " coexistence alternée » entre théorie et pratique}

Dès son premier recueil, Photography Against the Grain (1984), Sekula explique que, pour prendre la photographie à contre-courant, il lui a fallu passer par une "coexistence alternée » entre deux rôles : celui du «critique » qui théorise le médium; celui de «l'artiste visuel» qui le pratique (Sekula, 1984, 51). Le livre est d'ailleurs scindé en deux, les essais théoriques précédant les œuvres. Pour autant, il ne s'agit pas de proposer une « harmonie factice » entre solutions pratiques aux problèmes théoriques. Bien plutôt, ces deux modes d'énonciation différents ont vocation à se renforcer, à se nourrir, voire à se déstabiliser mutuellement. 
teur a défini sa pratique comme un « réalisme critique » : « un réalisme non pas des apparences ou des faits de société, mais celui de l'expérience quotidienne subordonnée ou opposée au capitalisme avancé " (Sekula, 1984, 52). De la même manière que sa pensée théorique a pris le contre-pied du modernisme, Sekula pense sa pratique à l'encontre du réalisme traditionnel - «tout réalisme est une réponse aux réalismes précédents ", écrit Jakobson (Sekula, 2012, 264). C'est là que l'articulation entre théorie et pratique est à chercher : la création ou le renouvellement des modes de narration et de captation du réel se fait en réaction aux failles mises en lumière dans les systèmes de représentation précédents.

Ayant théorisé la part essentielle de l'environnement culturel dans la portée sémantique de la photographie, Sekula ne pouvait suivre ces photographes qui, encouragés par l'institution muséale, « ont tendance à croire naïvement à l'efficacité de l'image unique " (Sekula, 1976-78, 155). Tout au contraire, ses installations se caractérisent par le recours à un large éventail de textes, destiné à une double mise à nue : l'éclairage des contraintes sociales et économiques, d'une part, et des structures prenant en charge le sens de l'autre. Là se situe « l'attrait majeur » de la photographie,

" son incontournable faculté à faire référence à la société, sa manière de décrire bien qu'en termes énigmatiques, réducteurs, trompeurs, et souvent superficiels un univers d'institutions sociales, de gestes, d'usages, de relations. Et le caractère problématique de ce pouvoir descriptif est en lui-même fascinant, à partir du moment où le monde vivant qui s'y manifeste est celui dont le photographe est d'emblée un acteur social, jamais un spectateur complètement innocent ou objectif. » (Sekula, 1984, 51)

Dès The Aerospace Folktales (1973), Sekula part donc de sa propre position sociale pour décrire le quotidien de son foyer familial alors que son père, cadre licencié d'une usine d'armement, est au chômage depuis de nombreux mois. L'œuvre comporte trois trames narratives distinctes: une série de cent quarante-deux images - tirages photographiques, documents administratifs, cartons noirs commentant la séquence à la manière des intertitres du cinéma muet - ; une bande-son de soixante-quinze minutes mixant quatre conversations entre l'auteur et ses parents ; un commentaire de l'auteur, affiché à la fin de la série. Ce «film désassemblé » illustre la contamination du foyer (sphère privée) par le complexe militaro-industriel (monde social): dans la bibliothèque des enfants, un livre sur la bombe atomique voisine avec les contes de Grimm, et la série est scandée par deux doubles pages montrant des corps mutilés ; au début, le portrait frontal du foyer est suivi du règlement intérieur, édicté par la compagnie; en fin de série, le $\mathrm{CV}$ du père rappelle que ce dernier est au chômage depuis deux ans et demi (figures 7-8). Appelé « l'ingénieur » dans la série, sa position se veut représentative d'une réalité sociologique nouvelle : la prolétarisation des cadres. Sekula le photographie s'adonnant à des tâches absurdes (le réglage acharné des lampes de la maison), incapable de reprendre sa vie en main dans une période d'oisiveté forcée. Pire encore, il ne parvient pas à « se penser historiquement ", à faire le lien entre sa propre situation et la guerre, et continue de chercher un emploi dans l'industrie aérospatiale, malgré ses connaissances sur l'arme nucléaire. 
Figure 7 : Sekula A. (1984), « The Aerospace Folktales » (1973), Photography Against a Grain, p. 118-119.

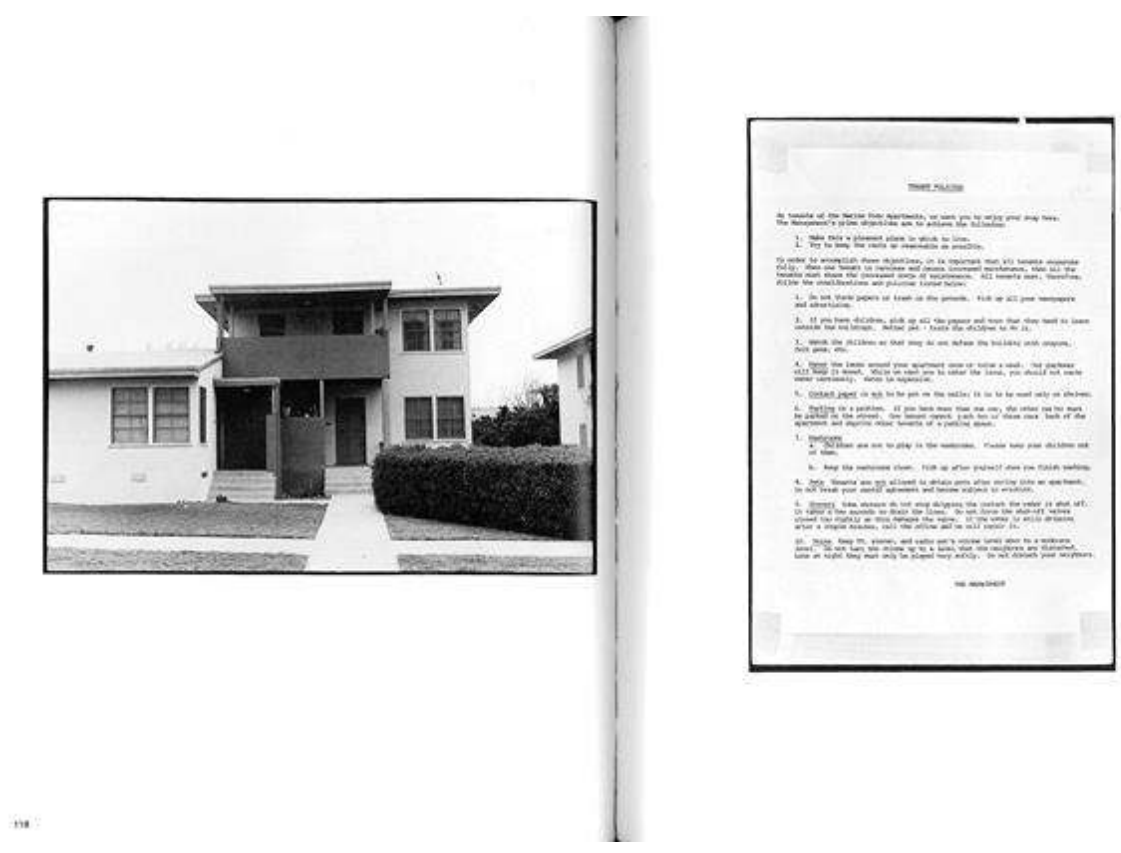

Figure 8 : Sekula A. (1984), « The Aerospace Folktales » (1973), Photography Against a Grain, p. $142-143$

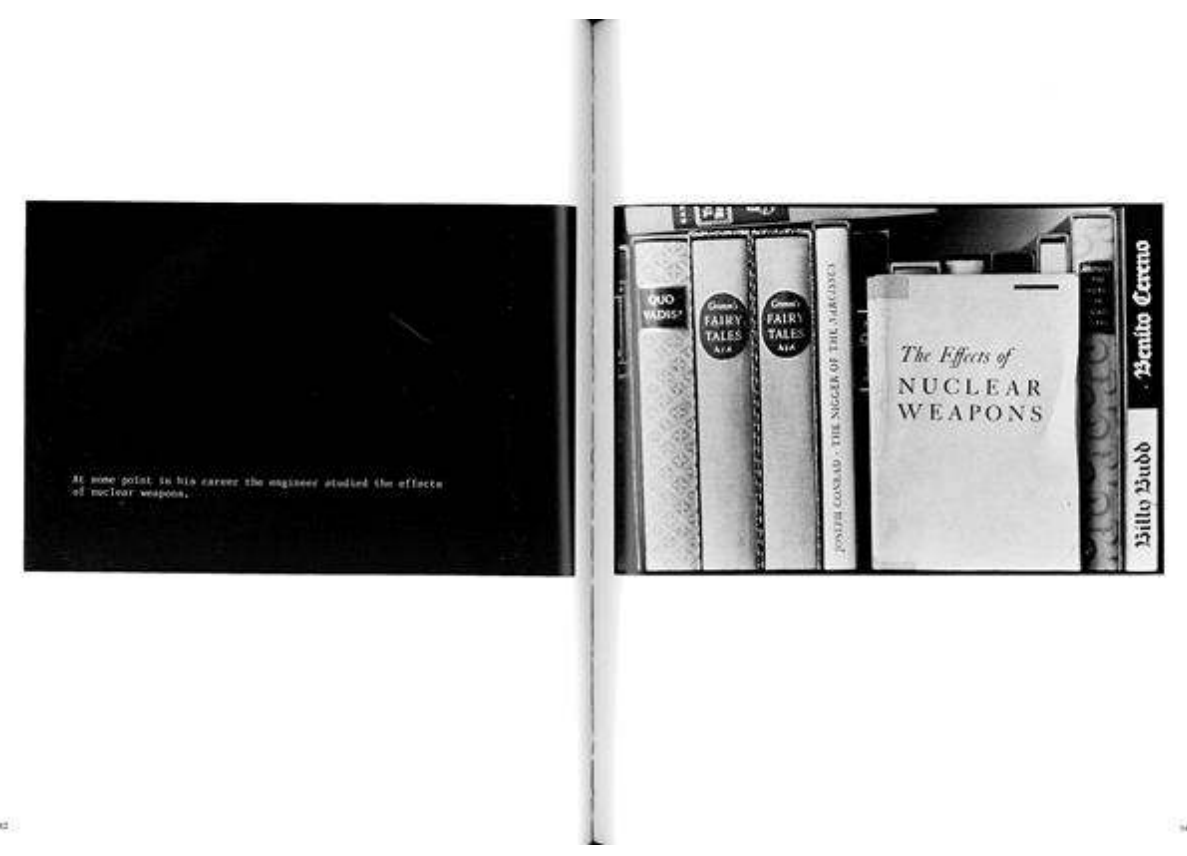

La série rappelle volontairement l'incapacité de la photographie à traduire la mainmise d'une idéologie sur un foyer. Le texte tire sa nécessité de cette conscience des manques propres au médium : «j'écris parce que le champ de représentation limité de l'appareil ne permet pas de photographier l'idéologie. Mais on peut faire une photo, reculer et dire : "regardez, dans cette photo, il y a de l'idéologie... entre ces deux photos, il y a de l'idéologie... et celle-ci se rapporte à celle-là". » (Sekula, 1973, 164). Pour cette raison, Sekula aime travailler avec un magnétophone. Alors qu'on imagine volontiers August Sander discuter avec ses modèles pour les inciter à trouver la pose la plus 
caractéristique de leur être social, la production du photographe allemand évacue délibérément cette préparation dialogique. À l'inverse, chez Sekula, la parole fait partie intégrante de l'installation : l'auteur fait entendre - par le biais de haut-parleur ou de cartons - la part active du dialogue entre l'artiste et son sujet, la photographie que l'on regarde apparaissant comme l'aboutissement d'une rencontre, d'un échange, voire d'un compromis entre les deux. Dans son œuvre, la parole est aussi un moyen de déstabiliser les hiérarchies allouant à chacun des capacités d'expression ${ }^{17}$.

Dans une telle démarche, la photographie articule pratiques privée et familiale, enquête sociale et documentaire. De fait, le réalisme de Sekula ne s'impose pas de style formel prédéfini : c'est la situation concrète qui sert de point de départ et qui impose à l'œuvre ses nécessités formelles. Il en va de même pour le montage entre l'image et le texte. Loin d'obéir à un quelconque esprit de système, la lettre doit offrir à l'image, non de la certitude, mais de la profondeur sociale : «les textes doivent établir, entre les images, des liens parfois inattendus mais plausibles» (Sekula, 2003, 107). En proposant des connexions nouvelles, les textes transforment l'instantané visuel en fait social, qui ne peut être séparé des conditions matérielles et idéologiques, visibles et invisibles, qui en sont à l'origine.

\section{2. La marge et l'attente : saisir l'événement par ses périphéries et ses conséquences}

31 Un trait récurrent de l'œuvre est le recours, dès ses débuts, à la séquence. Dans un dialogue avec le cinéma, Untitled Slide Sequence (1972) documente la sortie d'usine d'armement où travaillait son père, en pleine guerre du Vietnam, jusqu'à l'irruption impromptue des vigiles. Cette narration séquentielle met en évidence l'incomplétude inhérente à la photographie, contre le mythe du moment crucial et éphémère qui condenserait l'ensemble d'une réalité en une image. Mais la séquence peut aussi servir à traduire la répétitivité du quotidien, comme le père changeant une à une les ampoules du foyer, ou le caractère social du travail manuel, en montrant, d'une image à l'autre, les interactions entre les membres d'une équipe occupés sur une même tâche ${ }^{18}$.

Sekula s'intéresse aux temps faibles, à la vie ordinaire, au calme qui précède la tempête. Waiting for Tear Gas (1999) se focalise sur l'attente des manifestants, sur les visages révoltés et les corps concrets, mis en mouvement par des flux de capitaux abstraits. Corps fragiles, parfois délibérément nus, face à la menace annoncée des lacrymogènes, symbolisée par les rangs de CRS. La série est accompagnée d'une profession de foi qui illustre bien la position spatiale et théorique du photographe, son double refus du reportage et de la photographie d'art: "Il s'agissait de se laisser porter par le mouvement de révolte, de l'aube à trois heures du matin s'il le fallait, captant les accalmies, les attentes, les à-côtés de l'événement. Règles de base pour ce genre de reportage : pas de flash, pas de zoom, pas de masque à gaz, pas de carte de presse et pas d'envie de prendre à tout prix la photo poignante qui résumerait tout » (Sekula, 1999, $118)^{19}$. Une photo "embarquée", de la part d'un militant qui participe à la manifestation et la documente en même temps, du point de vue de la foule face aux forces de l'ordre. La pratique séquentielle permet ici de témoigner des longues heures qui précèdent l'affrontement, mais aussi de transformer la foule anonyme en une communauté d'individus, dotés de visages et d'affects (figure 9). 
Figure 9 : Sekula A. (1999), Allan Sekula, Waiting for Tear Gas, quatre-vingt-une photographies couleur et texte.

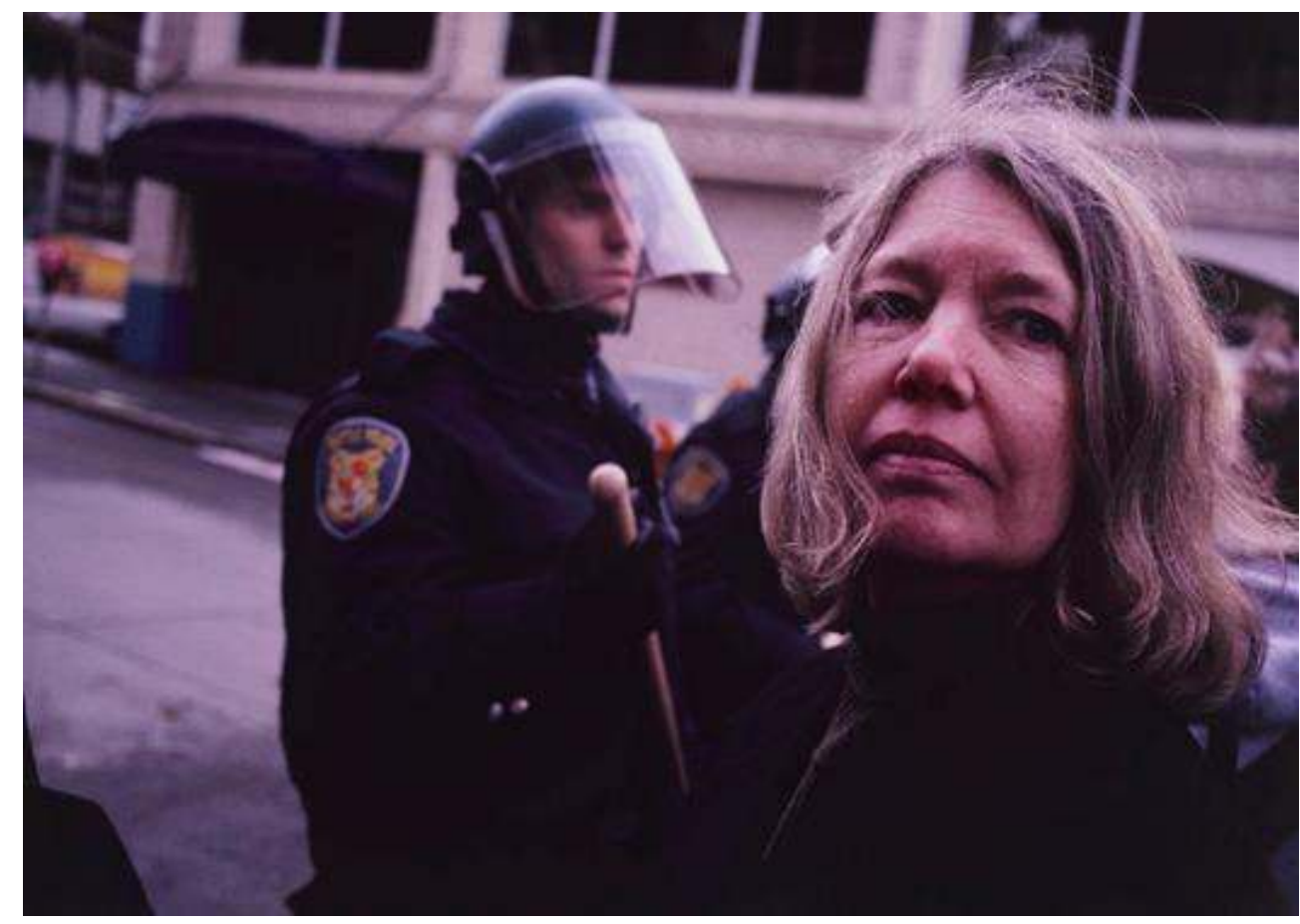

Dans «Défaire le modernisme", Sekula salue également le travail de son ami Fred Lonidier, à travers l'exposition The Health and Safety Game (1976-1978) : vingt panneaux, qui sont autant d'études de cas de travailleurs victimes de blessures ou de maladies professionnelles. À chaque fois, le titre du panneau fait référence à la partie du corps meurtrie: «Bras d'une emballeuse d'œufs» (figure 10) décrit le parcours d'une ouvrière et décompose la série d'événements qui a produit l'accident, manière de brosser un panorama non exhaustif des risques que le système productiviste fait courir à la santé des travailleurs. La photographie atteste de ces atteintes corporelles par des inserts cliniques qui enregistrent les chocs sur les corps. Mais le travailleur lui-même n'est pas réduit à un corps passif, puisqu'apparaissent aussi des extraits de son témoignage, mêlant expérience du travail et lutte pour se faire dédommager, agrémenté parfois de textes juridiques ou de minutes de procès. "Un véritable documentaire critique social cadrera le crime, le procès, et le système de justice avec ses mythes officiels ", écrivait à ce propos Sekula $(1976-78,149)^{20}$.

On pourrait aussi penser à un film militant du Groupe Medvedkine, Nouvelle Société $N^{\circ} 7$ (1969). À la veille de Noël, un jeune OS perd une main sous une presse, sous l'autorité d'un contremaitre qui le somme d'accélérer la cadence. Un long récit en voix off raconte les circonstances de l'accident, l'obligation de courir après le temps qui fatigue l'attention et le chef qui se moque de la sécurité. Au lieu de faire de cet accident un événement ponctuel, le commentaire prend soin de remonter l'enchainement des causes qui aboutit nécessairement au drame. Des photographies d'OS, prises illégalement à l'atelier, accompagnent le témoignage, faisant peser le risque sur chacun d'eux. 
Figure 10 : Lonidier F. (1976), « Bras d'une emballeuse d'œufs », The Health and Safety Game, vingt panneaux cartonnés avec photographies et texte.

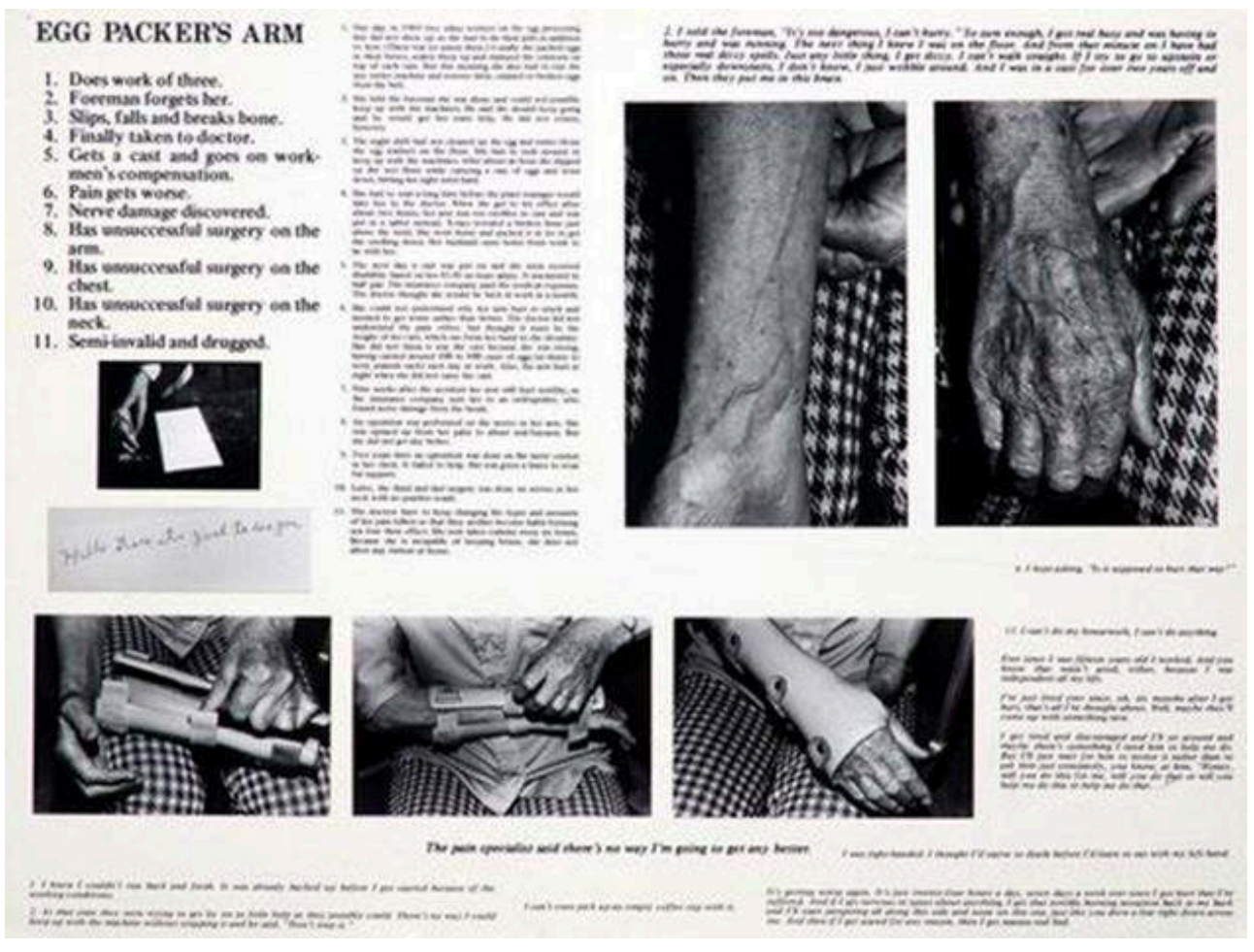

\section{3. Le musée des travailleurs anonymes: Ship of fools et The Dockers' Museum}

35 À partir de la fin des années 1980, Sekula se consacre de plus en plus au commerce maritime mondial, ses infrastructures portuaires et les allers-retours de ses cargos ( $95 \%$ des marchandises vendues dans le monde transitent par cargo). Fidèle à son matérialisme, l'espace maritime apparaît comme le support fluide et concret d'un libreéchange technicisé et dérégulé, entre des espaces aux fonctionnements divisés et aux développements inégaux. Son travail s'attaque à deux grands mythes du néolibéralisme : le devenir immatériel du capitalisme et la mondialisation heureuse.

Mélange de recherche et de création, à la manière du cinéma de Harun Farocki, Fish Story (1995) a été pensé pour exister sous forme d'exposition et de publication. Des essais et photographies de Sekula côtoient des récits et anecdotes d'ouvriers de la mer que l'auteur a glanés à travers le monde, après l'effondrement du bloc soviétique, et au moment où la Corée connaît un boom économique important. Souvent, ses photographies isolent des travailleurs dans une tâche manuelle, soudant des pièces, récoltant du cuivre ou levant une affiche de film - même les images semblent avoir un poids ici (figures 11-12). Ces portraits à la lisibilité quasi pédagogique affrontent les fantasmes des intellectuels de tout bord qui pensent l'économie moderne comme un capitalisme de flux, basé sur la collecte de données - Sekula parle d' " oubli de la mer » chez les élites. Or, le moteur de ce commerce international, aussi essentiel qu'anachronique, reste le travail humain, grand oublié de l'imaginaire contemporain : "Si le marché boursier est le lieu où règne le caractère abstrait de la monnaie, le port est le lieu où les biens matériels apparaissent dans toute leur masse, dans le flux réel 
des échanges ", écrivait Marx, repris par Sekula $(1995,4)$. Sans ces hommes et ces femmes qui construisent, réparent, entretiennent, conduisent, chargent et déchargent les bateaux, les flux de marchandises s'arrêteraient net.

Figure 11 : Sekula A. (1995), « Bo'sun conduisant le treuil avant. Mouillage au Terminal ECT/Sea Land. Maasvalakte, Port de Rotterdam », Fish Story, p. 69.

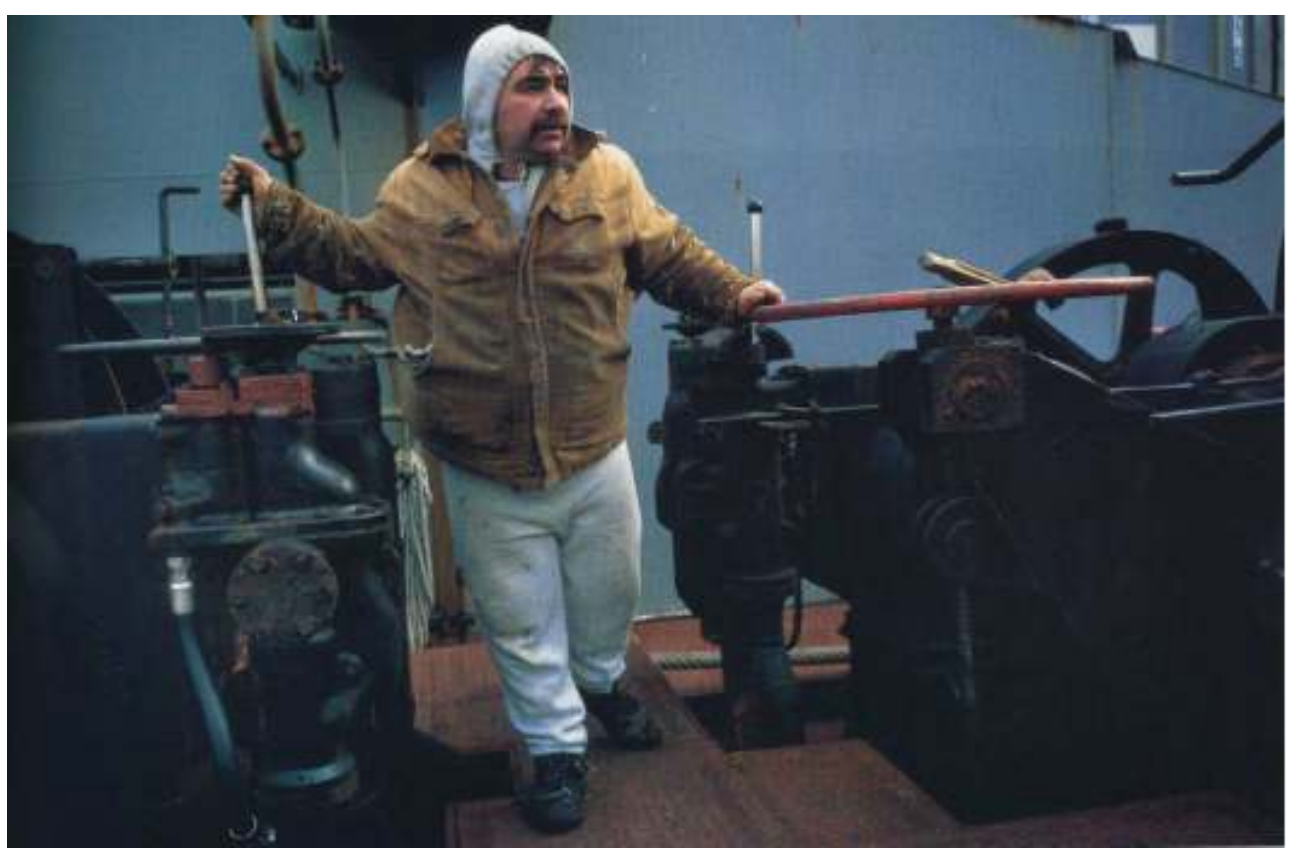

Figure 12 : Sekula A. (1995), "Ouvrier installant une affiche de cinéma. Séoul », Fish Story, p. 98.

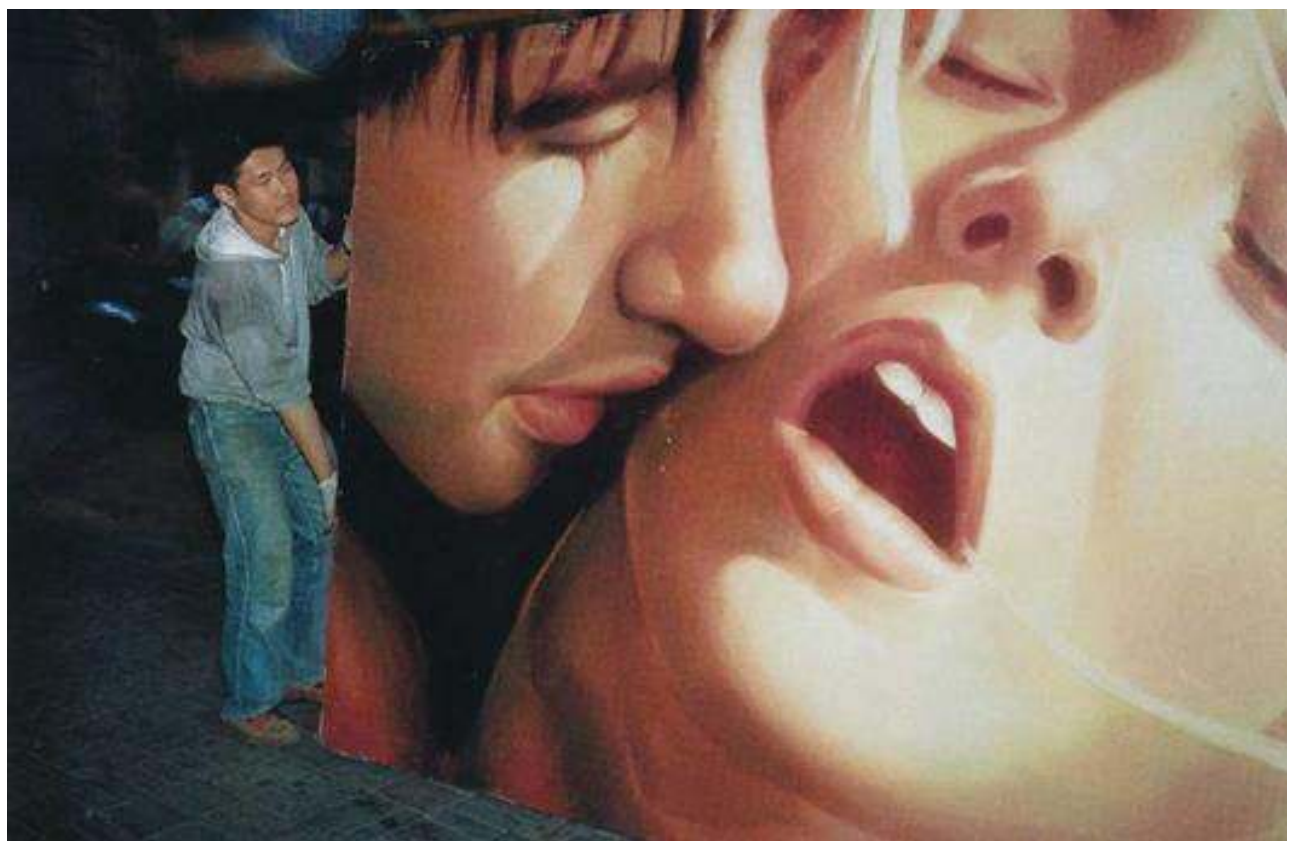

La cause de cette "cécité cognitive " a été l'invention du conteneur, qui a largement contribué à effacer de l'imaginaire collectif le transport des marchandises. Forgotten Space (2011), documentaire réalisé avec Noël Burch, s'inscrit dans une démarche de contre-information, en suivant le trajet de ces boîtes autour du globe. Si elle a permis 
de réduire drastiquement la manutention et le temps de chargement, et donc d'accélérer la circulation des marchandises à travers le monde, la conteneurisation massive a aussi fait perdre aux dockers internationaux une grande part de leur maitrise sur les opérations de chargement et de déchargement, ce qui a permis aux entreprises de réduire sans cesse les coûts de main d'œuvre en se délocalisant à travers le globe. Dans ce film, Sekula dévie régulièrement de sa route pour interroger les exploités ou les laissés pour compte du libre-échange, avec ce constat désarmant : qu'ils soient dans le cycle des échanges ou qu'ils en aient été éjectés, les prolétaires de tous les littoraux semblent partager une précarité similaire.

C'est là une autre ligne de force de ce travail documentaire: tenir ensemble le développement inégal et interdépendant du capitalisme mondialisé, et renouer les liens de causalité entre les pôles que la médiatisation des échanges tend à séparer. Waiting for Tear Gas rappelle la solidarité des hommes et des femmes des docks avec les altermondialistes, parce qu'ils ont conscience qu'au-delà des mers, il y a «d'autres corps qui font le même travail et que le commerce mondial ne se réduit pas à un clic de souris » (Sekula, 1999, 119). En mettant en concurrence les ouvriers qualifiés des pays développés, protégés par des droits sociaux conquis de haute lutte, avec des paysans asiatiques ou africains à la limite de l'esclavage, le néolibéralisme produit de la misère des deux côtés de la chaîne ${ }^{21}$. "Se peut-il que ces boîtes, comble de l'ordre, de l'efficacité et du progrès, engendrent désordre et destruction, et déséquilibrent le monde? " , s'interroge le commentaire de Forgotten Space.

Avec la conteneurisation et le chômage, ce qui disparaît, c'est aussi la culture commune au monde ouvrier, et plus spécifiquement aux habitants des ports. Lecteur de Moby Dick, C. L. R. James offre à Sekula une compréhension matérialiste du grand œuvre de Melville ${ }^{22}$. Socialiste antillais, victime du maccarthysme, James souligne que l'équipage multiracial du baleinier était composé avant tout de travailleurs, reliés entre eux par une « culture quotidienne du lieu de travail [qui] fournit la base de la solidarité et de la révolte » (Sekula, 1995, 72). En 1999, Sekula effectue un voyage autour du monde sur le Global Mariner, un ancien cargo transformé en musée itinérant par l'International Transport Worker's Federation (ITF) pour alerter de port en port sur les conditions de travail en mer. Ce navire propose un contre-usage de la mer: en lieu et place de la concurrence sauvage entre tous, il sert au rapprochement et à la coopération entre les peuples. Une sorte d'utopie, proche de la République universelle imaginée par James, et avant lui par les anarcho-syndicalistes des années 1920, qui rêvaient à « une dictature ouvrière sur toutes les mers du monde ", soit un réseau révolutionnaire qui, opposé au réseau mondial du commerce, relierait entre eux les travailleurs comme sujets.

Après son expérience sur ce bateau-musée, le dernier grand projet de Sekula sera une exposition fluctuante de ses propres collections: The Dockers' Museum. En plus de ses photographies y voisinent de nombreux « objets d'intérêt ", des artefacts vernaculaires achetés sur Internet ou lors de brocantes. Sekula se réfère à Eduard Fuchs et à son histoire de la culture de masse, à qui Benjamin a rendu hommage : « dans les musées, notait Fuchs, la culture se donne à voir dans son costume d'apparat, rarement dans son habit de tous les jours, qui est le plus souvent pauvre» (cité par Benjamin, 1937, 220). Fuchs imagine une collection d'objets anonymes, délivrant la culture du marché de l'art tout en donnant à percevoir « la manière dont le monde et les choses apparaissent à la communauté " (cité par Benjamin, 1937, 223). De la même manière, Sekula a accumulé de façon quasi obsessionnelle des objets fabriqués en série, à travers lesquels le travail 
maritime apparaît aux yeux du monde : des cartes postales ou des gravures du début du $\mathrm{XX}^{\mathrm{e}}$ siècle, des figurines de dockers de Macao ou des reproductions de sculptures du Belge Constantin Meunier. Le but avoué est de «retrouver l'acte primordial de collectionner dans toute sa force critique " (Sekula, 2013, 116) : à la différence de l'archive qui vise la quantité et le classement, souvent à des fins de pouvoir, le collectionneur opère en amont une sélection rigoureuse d'un très petit nombre d'objets, afin de garder une cohérence, tout en neutralisant les hiérarchies et les normes culturelles. Il entend ainsi cartographier un domaine d'images alternatif, qui rendrait sensible la vie des travailleurs de la mer.

41 Tout y est exposé sur un pied d'égalité, sans que ses propres images ne tiennent le rôle principal: le spectateur fait face à des signes énigmatiques laissés à sa libre interprétation, à son propre montage. De nombreuses lectures sont possibles. L'une d'elles suggère le passage d'un travail de force, sujet de fantasme, à une industrialisation des ports : en effet, la séquence Sugar Gang (Santos) entre en résonance avec une carte postale publicitaire de 1908, "L'embarque du café », où les dockers du port brésilien posent en ligne avec des sacs de café sur la tête. Sur la carte postale, un porteur est mis en avant, comme une attraction spectaculaire, chargé de quatre $\operatorname{sacs}^{23}$; ce caractère spectaculaire revient dans une photo de tournage du film Rio, rythme d'amour (Simon S. S., 1946), où des débardeurs d'opérette sont joués par des danseurs au déhanché appuyé ; un troisième objet montre une tasse en forme de tête de docker, aux traits caricaturaux, que Sekula a recouvert de sacs de café. Cette juxtaposition témoigne du fonctionnement de cette économie de l'imaginaire évoquée plus haut, où la force de travail est transformée en spectacle et vidée de sa réalité à force d'être fantasmée. À l'inverse, les ouvriers photographiés par Sekula usent de techniques de levage élaborées, mais ont disparu de la culture de masse. Une colonne vertébrale synthétique trône au milieu de la salle, comme pour rendre plus sensible encore le poids réel des sacs sur le dos des travailleurs (figures 13-16). Pareille comparaison opère tant sur le plan du travail lui-même, que sur sa médiatisation : «Le conflit de classe n'est pas qu'une question économique et politique; c'est aussi un conflit de représentations. » (Sekula, 1983, en ligne)

Une telle manière d'envisager l'espace muséal prend également à contre-courant la conception du spectateur qui était celle du modernisme. À l'encontre du documentaire, ce courant supposait un « régime d'exception des arts plastiques» (Sekula, 2012, 266) à même d'attirer le regardant dans une relation d'absorption contemplative ${ }^{24}$. Tout au contraire, Sekula, toujours fidèle à Marx, revendique une photographie qui ne se contenterait pas d'enregistrer le réel, mais s'emploierait à le transformer. Très concrètement, cela passe par le choix des lieux d'exposition. Afin d'atteindre des publics qui ne sont pas familiers de l'espace muséal, Lonidier exposait ses panneaux dans des locaux syndicaux; Sekula, suivant l'exemple du Global Mariner, a exposé dans de nombreux ports, de New York à Anvers, en passant bien sûr par Santos. Cela passe ensuite par la capacité du documentaire à donner à imaginer des alternatives crédibles au néolibéralisme : des œuvres qui aident à poser les termes du débat et tentent d'être force de proposition (Sekula, 2012, 267). 
Figure 13: Anonyme (v. 1908), « L'embarque du café » [titre donné par Allan Sekula], carte postale, achetée par Sekula sur eBay le 9 mai 2010 /

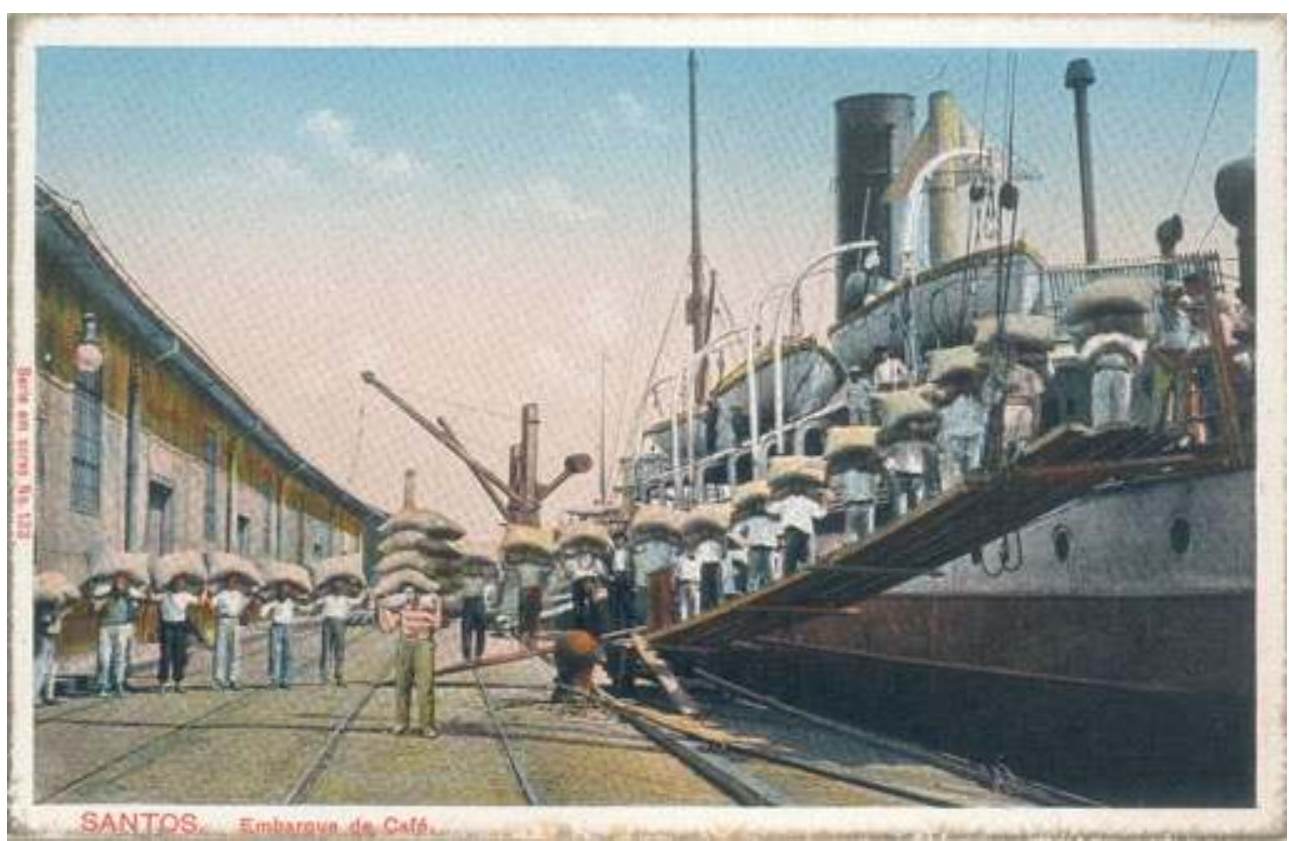

Figure 14 : Van Pelt H. (v. 1946), Photographie de tournage du film Rio, Rythme d'amour, noir et blanc, achetée par Sekula sur eBay le 6 mai 2010.

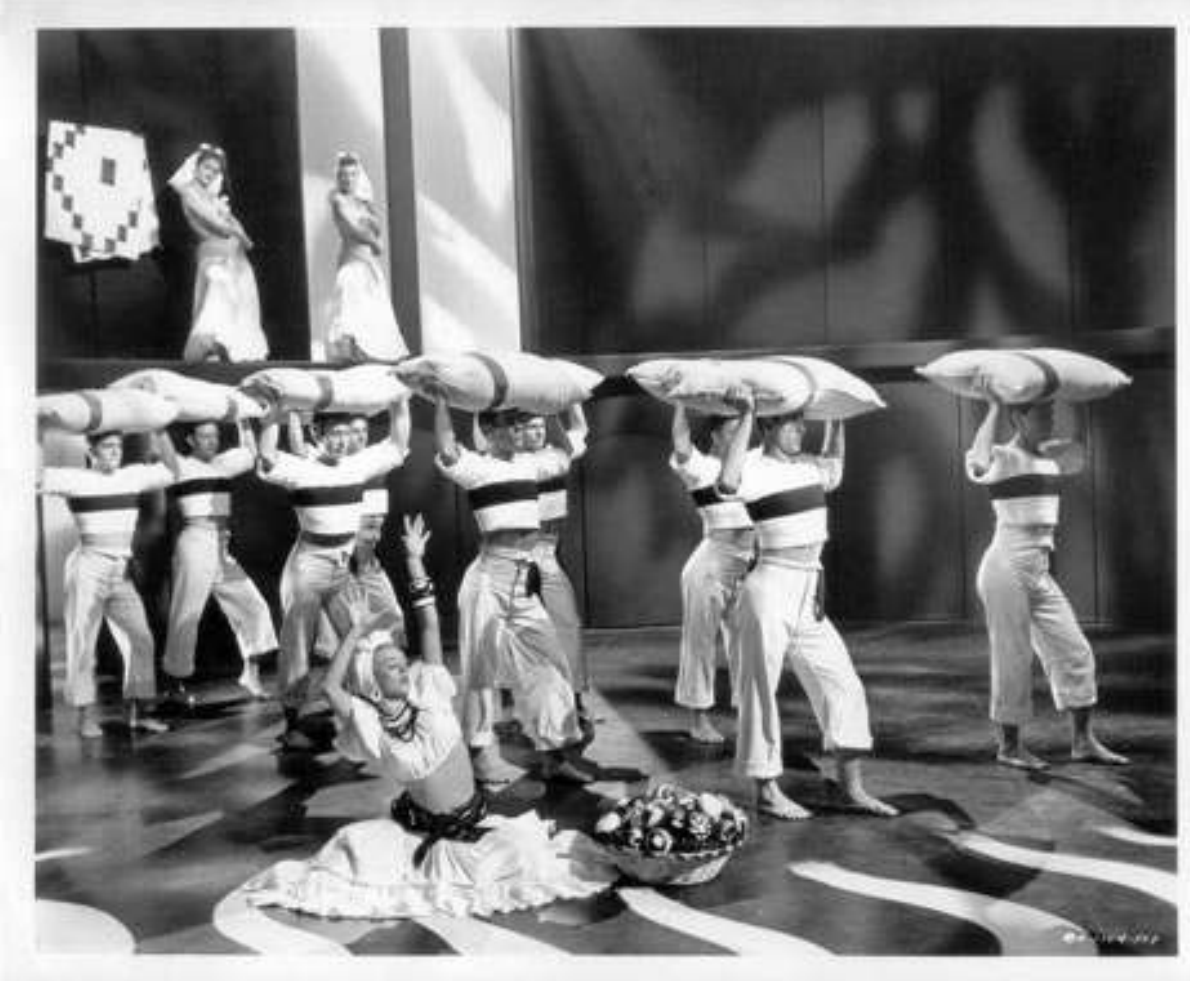


Figure 15 : Sekula A., The Dockers Museum (détail d'une salle)

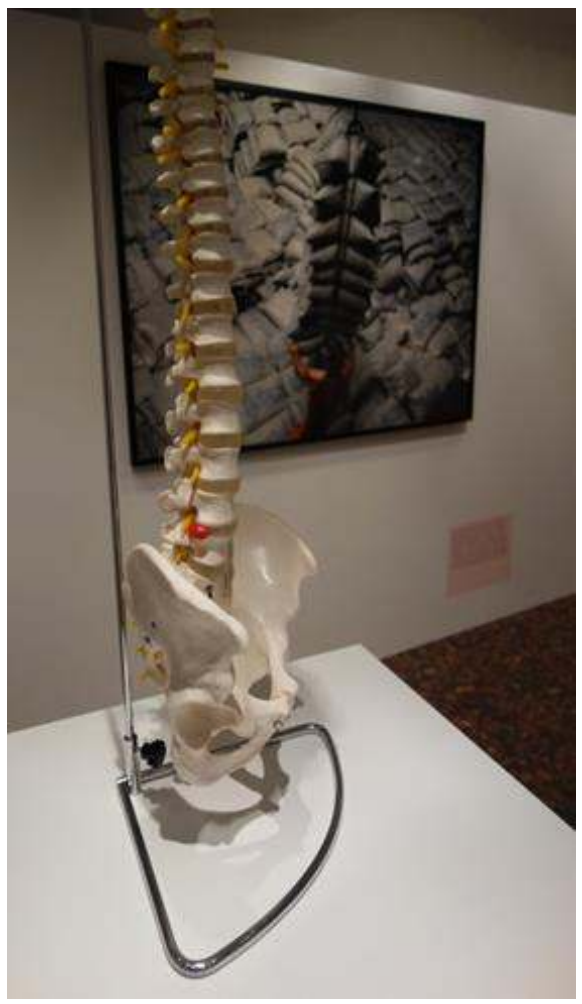

Figure 16 : Sekula A. (2010), Working (Santos), photographie couleur.

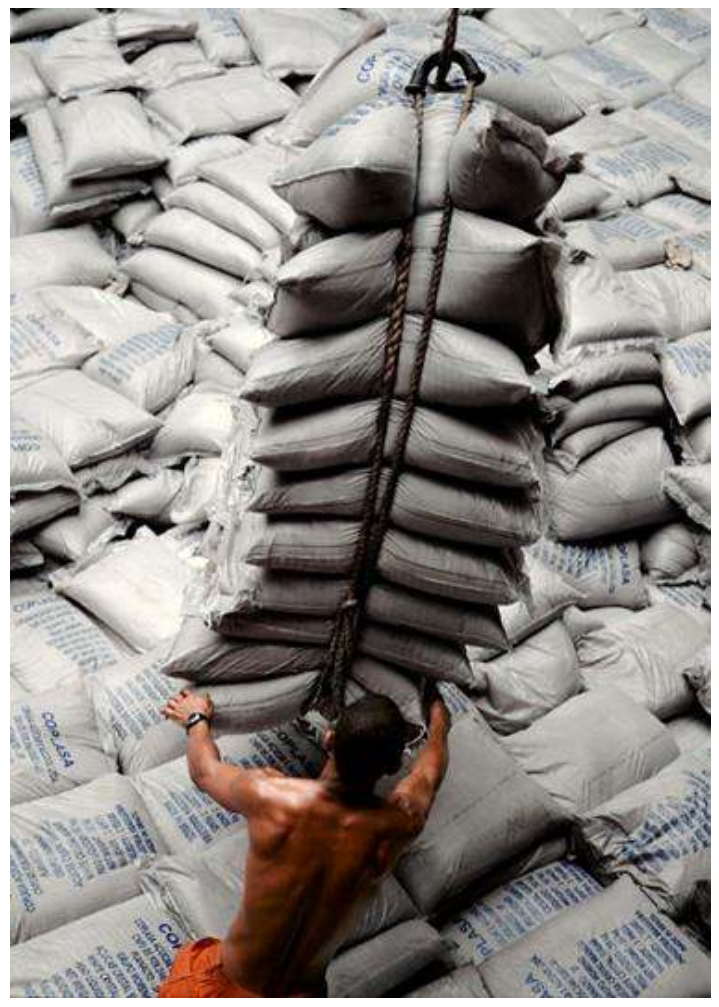




\section{Conclusion} effet, Le Facteur humain (2011) ${ }^{25}$, montage de bandes institutionnelles et publicitaires, fait entendre les réflexions d'un jeune ingénieur enthousiaste glissant de l'émerveillement devant les principes du taylorisme, jusqu'au doute quant à leur application réelle. L'intérêt du travail de Le Texier est de faire résonner les images institutionnelles avec les idéologies qui les produisent, de façon à faire éclater les contradictions qu'elles sont censées recouvrir : la recherche de l'efficacité s'étend à l'ensemble de la société (usine bien sûr, mais aussi loisirs, consommation, jeux enfantins, univers domestique, agriculture, domaine militaire...), au risque de mettre en péril la sacro-sainte liberté individuelle. Le film prolonge l'essai sur l'émergence du langage visuel du capitalisme, en mobilisant les mêmes références (Taylor, Gilbreth), mais en s'attachant cette fois à la propagande filmique institutionnelle, là où Sekula compulsait la littérature d'entreprise, et qui façonne à son tour une économie de l'imaginaire.

Comme Sekula encore, Le Texier insiste sur l'interconnexion entre le travail et le foyer, à travers la correspondance de l'ingénieur avec son épouse: tandis que celui-ci réorganise la production, cette dernière cherche à rationaliser ses tâches domestiques, signe d'une contamination idéologique, mais aussi économique, car «ce qui est bon pour l'usine est bon pour la cuisine », la maison faisant partie intégrante du système 
industriel. Dans ce dialogue réside en revanche une différence notoire entre les deux artistes, puisque le cinéaste-sociologue adopte, par le biais d'une relation épistolaire fictive, mais dûment documentée, le point de vue de l'ingénieur. Il peut alors imaginer les affects qui animaient cette classe intermédiaire chargée de collecter du savoir sur le travail afin d'augmenter la production. Le parti pris est de montrer une trajectoire mentale : l'idéal abstrait du jeune diplômé - amélioration rationnelle des conditions de travail et des salaires menant à la paix sociale - se fracasse sur la réalité humaine, et l'obsession managériale pour l'observation, la surveillance, la mesure et la mise en fiche du travail finit par produire le pire. Au lieu de l'efficience attendue, c'est une grève dure qui est déclenchée par des ouvriers mal notés. Et l'ingénieur, peu à peu transformé en machine, ne peut que conclure : «plus j'analyse le travail, et moins je le comprends».

Le travail, activité humaine et sociale par excellence, échappe à l'analyse productiviste et à la raison managériale. Leslie Shedden lui-même a également produit le contrepoint parfait aux commandes précises et orientées de Dosco: une photo de groupe où les mineurs posent souriants face à l'objectif (figure 17). Dans un tel portrait, les corps sont délivrés de l'activité laborieuse, tout en conservant la cohésion du collectif de travail. Lors de ses visites à Glace Bay au début des années quatre-vingt, Allan Sekula a été marqué par la « continuité culturelle »: la préservation d'une culture ouvrière malgré la fermeture du dernier puits quinze ans auparavant. Et l'auteur d'en déduire " qu'on n'en finit pas facilement avec la civilisation de la classe ouvrière ${ }^{26}$.

Figure 17 : Leslie Shedden, Groupe de mineurs après la remontée, non datée, archives Shedden.

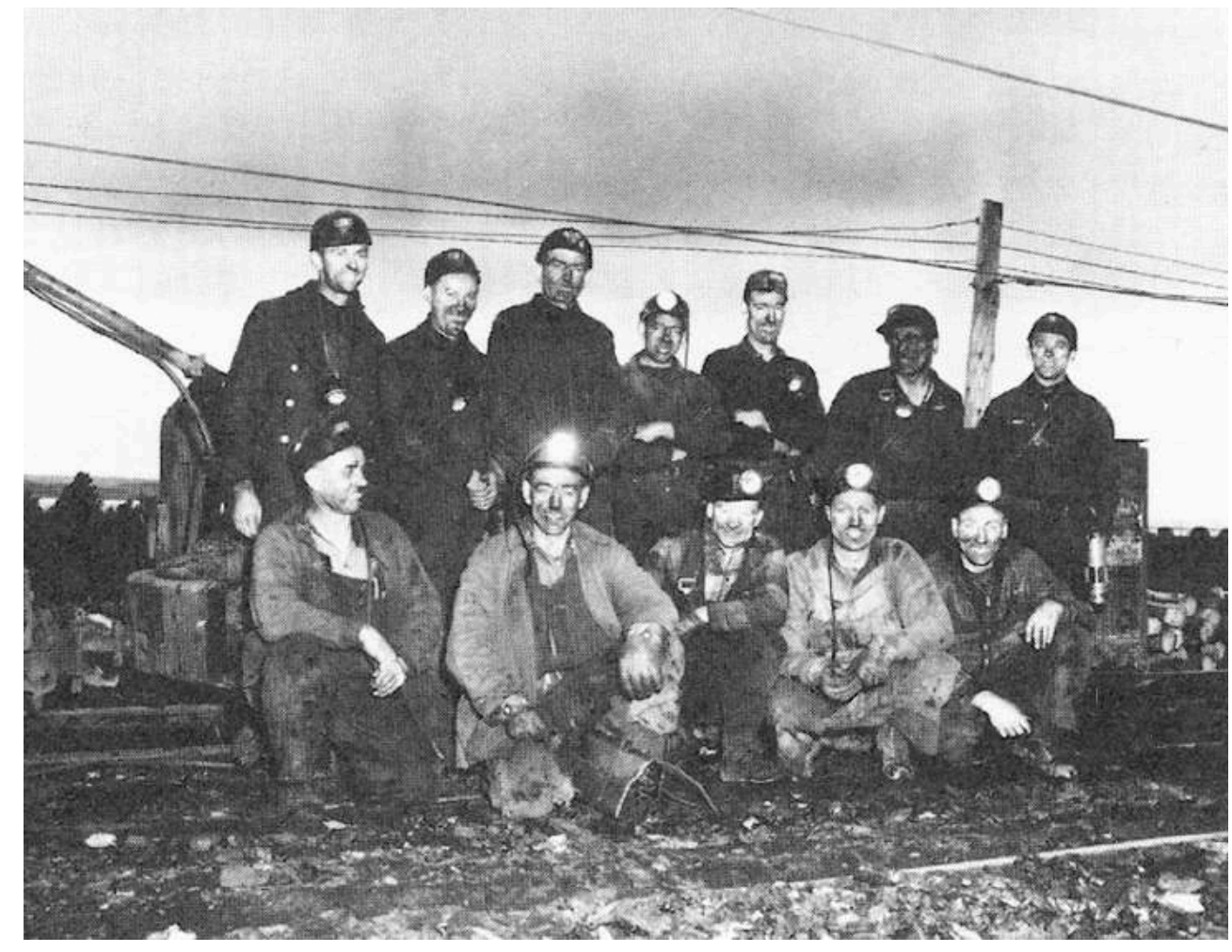




\section{BIBLIOGRAPHIE}

Agricola G. (1556) De Re Metallica, H. Froben et N. Episcopius, Bâle. The Mining Magazine, London, 1912, Traduction du latin vers l'anglais par Herbert et Lou Hoover.

Bakthine M (1977) Le Marxisme et la philosophie du langage. Essai d'application de la méthode sociologique en linguistique, Paris, Minuit.

Barthes R. (1970) [1957] « La grande famille des Hommes », Mythologies, Paris, Seuil, « Points ». Barthes R. (1972) «Les planches de l'Encyclopédie », Nouveaux essais critiques, Paris, Seuil.

Benjamin W. (2006) [1937] « Eduard Fuchs », Euvres III, Traduit par Maurice de Gandillac, Paris, Gallimard, « Folio essais ».

Benjamin W. (2006) [1940] « Thèses sur le concept d'histoire », Euvres III, Traduit par Maurice de Gandillac, Paris, Gallimard, « Folio essais ».

Bourke-White M. (1931) Série "Hard Coal", Fortune, Février 1931.

Engels F. et Marx K. (1845) L'Idéologie allemande, UQAC, [en ligne].

Galassi P. (1981) Before Photography, New York, MoMa.

Garçon A.-F. (2010) « Pratique, technique, technologie ? ", ArcheoSciences, n 34, p. 121-126.

Le Tallec A. (2015) « Quand l'exégèse d'un texte lui confère le statut de manifeste. L'exemple du Nouveau Documentaire Social », Marges, $\mathrm{n}^{\circ}$ 21, p. 35-46.

Piron S. (2018) L’Occupation du monde, Bruxelles, Zones sensibles.

Rancière J. (1995) La Mésentente, Paris, Galilée.

Rancière J. (2008) Le spectateur émancipé, Paris, La Fabrique.

Renault M. (2016) C. L. R. James. La vie révolutionnaire d'un « Platon noir », Paris, La Découverte.

Root M. A. (1864) The Camera and the Pencil, Philadelphia, Root.

Szarkowski J. (1973) Looking at photographs. 100 Pictures from the Collection of the Museum of Modern Art, New York, MoMa.

Sekula A. (1973) « The Aerospace Folktales », in Photography against the Grain. Essays and Photo Works (1973-1983), Halifax, The Press of the Nova Scotia College of Art and Design, 1984, p. 105-164.

Sekula A. (1983) «Photography between Labor and Capital », in Benjamin Buchloh, Robert Wilkie, Mining Photographs and Other Pictures, 1948-1968, A Selection from the Negative Archives of Shedden Studio, Glace Bay, Cape Breton, Cape Breton, The University College of Cape Breton Press. Nous avons publié pour la revue en ligne Débordements (www.debordements.fr) une traduction en cinq parties de cet essai.

Sekula A. (2013) [1974] « Sur l'invention du sens en photographie », in Écrits sur la photographie, Paris, Beaux-Arts de Paris éditions. Traduit de l'anglais par Marie Muracciole, p. 61-98.

Sekula A. (2013) [1976-78] « Défaire le modernisme, réinventer le documentaire : notes sur une politique de la représentation », Écrits sur la photographie, op. cit., p. 143-174.

Sekula A. (2013) [1984] « La photographie à contre-courant. Une introduction », Écrits sur la photographie, op. cit., p. 47-60. 
Sekula A. (2013) [1986] « Trafics dans la photographie », Écrits sur la photographie, op. cit., p. $175-220$.

Sekula A. (2018) [1995] Fish Story, MACK.

Sekula A. (1996) « An Eternal Esthetics of Laborious Gestures », Grey Room, n 55, Spring 2014, p. 16-27.

Sekula A. (1999) « Waiting for tear Gas », Multitudes, $n^{\circ}$ 7, 2001, p. 118-129.

Sekula A. (2003) « Entre les mailles du Net et la grande bleue », in Titanic's Wake, Le Point du Jour. Sekula A. et Burch N. (2011) The Forgotten Space, DVD Wild Art Film.

Sekula A. (2012) «Onze remarques et une question sur le documentaire », in Chantal Pontbriand (dir.), Mutations, Perspectives sur la Photographie, Steidl/Paris Photo.

Taylor F. W. (1903) « Le management de l'atelier », in Harwood Merrill (1970), Les Classiques du management, p. 63-71.

Day G. (2015) «La poétique transitive d'Allan Sekula : métonymie et métaphore dans Lottery of the Sea, Ship of Fools et The Dockers' Museum », in Van Gelder H. (dir.), Ship of Fools / The Dockers Museum Rennes, La Criée et le Frac Bretagne, p. 57-71.

Weil S. (1951) La Condition ouvrière, Paris, Gallimard, « Idées ».

Woronoff D. (1994) Histoire de l'industrie en France du XVI ${ }^{e}$ siècle à nos jours, Paris, Seuil, « Points Histoire ", 1998.

\section{NOTES}

1. L'exposition, visible du 6 avril au 20 mai 2012, comportait des œuvres de la collection du Frac Bretagne, et a donné lieu à la publication du livre Ship of Fools / The Dockers' Museum (2013), sous la direction de Hilde Van Gelder.

2. Sur la visée « manifestaire » du texte, voir Le Tallec A. (2015).

3. Exposition au Museum of Modern Art de New York, du 28 février au 7 mai 1967.

4. Dans son best-seller Looking at Photographs : 100 Pictures from the Collection of the Museum of Modern Art, Szarkowski affirme n'avoir regroupé ses cent photos préférées que pour le plaisir de l'œil et la délectation du lecteur (Szarkowski, 1973, 9).

5. La version livresque de Fish Story comporte un long essai en deux parties intitulé «Dismal science " (morne savoir).

6. Sekula étudie différents aspects de cette exposition dans trois textes au moins $(1986,1996$, 2003). Dans Trafics dans la photographie, il rappelle que The Family of Man est installée au Guatemala quelques mois à peine après un coup d'État contre le président socialiste Jacobo Arbenz, démocratiquement élu, et renversé avec le soutien de l'armée américaine.

7. Cf. Sekula, 1996. L'exposition, qui compte cinq-cents photos de soixante-treize auteurs différents, a voyagé dans trente-sept pays sur les six continents entre 1955 et 1964 et a rassemblé plus de neuf millions de visiteurs. Son catalogue s'est vendu à quatre millions d'exemplaires.

8. La traduction anglaise, " against the grain ", apparait dans le titre du premier recueil de textes de Sekula, expression qui a été retraduite en français par «à contre-courant ».

9. Leslie Shedden reprend en 1948 le studio que son père avait ouvert à Glace Bay trente ans plus tôt. Petit commerçant sans ambition artistique, mais doté d'un solide savoir-faire technique, il se spécialise dans le portrait et immortalise avec un grand respect les événements importants de la vie familiale des mineurs et employés de sa ville. Néanmoins, son client le plus lucratif demeure 
l'entreprise locale, Dosco, et son studio ne survivra pas à la faillite de cette dernière, en 1967. Sekula rencontre le photographe à l'occasion de l'écriture de cet essai et le cite à plusieurs reprises.

10. C'est oublier que la production appartient aux corporations, et que ces dernières imposent à la fois les procès du travail et le secret professionnel à leurs membres. C'est oublier, surtout, que le savoir artisanal est un savoir empirique, appris sur le tas, par des maximes, par la connaissance sensible des outils et de la matière, et par la reproduction des gestes des pairs, durant des années d'apprentissage.

11. Il s'agit de la Midvale Steel Company, une aciérie de Pennsylvanie.

12. Sekula ajoute que "dans l'atelier mécanisé, c'était la carte d'instruction qui adressait aux ouvriers un plan de travail prédéterminé [...] à partir d'une liste de mouvements séquentiels, quantifiés et analysés. » Ainsi, la dégradation des conditions de travail s'accompagne d'une dégradation des représentations de ce dernier, au sein même de l'atelier.

13. «Pour copier les millions et millions de hiéroglyphes qui couvrent, même à l'extérieur, les grands monuments de Thèbes, de Memphis, de Karnak, etc., il faudrait des vingtaines d'années et des légions de dessinateurs. Avec le daguerréotype, un seul homme pourrait mener à bonne fin cet immense travail. (...) Les images photographiques étant soumises dans leur formation aux règles de la géométrie, permettront, à l'aide d'un petit nombre de données, de remonter aux dimensions exactes des parties les plus élevées ; les plus inaccessibles des édifices. » (Arago, 1839, cité par Sekula, 1983)

14. Sylvain Piron rappelle qu'en droit romain « la catégorie d'occupatio désigne le titre légitime accordé au premier occupant d'une terre. » De fait, « les “terres occupées” marquent l'extension du monde pacifié. » Ce principe juridique servira encore à établir la souveraineté territoriale des colons et leur appropriation des terres du Nouveau Monde (Piron, 2018, 15).

15. À la fin des années 1870 , les grands dépôts des Chemins de fer du Nord se targuent d'avoir augmenté le rendement ouvrier de $25 \%$ en passant à la lampe électrique.

16. L'éditeur commande à Kollar deux mille clichés pour une encyclopédie photographique en quinze tomes, dédiés à diverses branches de l'industrie nationale : La France travaille (1930-1932). Les photographies sont accompagnées de texte d'écrivains et journalistes de l'époque. Le cliché examiné appartient au chapitre "L'Automobile, la route ", sous la plume de Hervé Lauwick, publié dans le tome II de l'édition intégrale.

17. On peut sans doute voir ici une influence de Jacques Rancière, dont la lecture, notamment du Maître ignorant, a marqué Sekula. Régulièrement dans son œuvre, Sekula s'emploie à défaire ce que le philosophe a appelé un "partage du sensible »: " un ordre du visible et du dicible qui fait que telle activité est visible et que telle autre ne l'est pas, que telle parole est entendue comme du discours et telle autre comme du bruit » (Rancière, 1995, 52). Dans le même ordre d'idée, l'auteur ne cache pas son admiration pour L'Histoire orale de la Grande Dépression, de Studs Terkel (1970), où le journaliste donne la parole aux vaincus de l'ordre économique capitaliste.

18. On songe ici au triptyque « Dockers chargeant un cargo sucrier à Calais » publié dans Calais vu par Allan Sekula (1996, 22), auquel fera écho plus tard Sugar Gang (Santos) (2010). Dans ces deux œuvres sérielles, des débardeurs chargent et déchargent des sacs de sucre, et les séries font apparaître le travail tout à la fois comme épreuve physique (des corps pliés sous le poids des marchandises) et comme activité sociale (la communication entre les hommes, la présence d'une autorité hiérarchique).

19. L'œuvre consiste en un diaporama de quatre-vingt-une photographies couleur, accompagné d'un texte, publié plus tard dans la revue Multitudes.

20. La métaphore pénale renvoie au mot de Benjamin, voyant dans les photos parisiennes d'Eugène Atget des « scènes de crime ». 
21. L'installation photographique From Freeway to China (Version 2, for Liverpool) (1998-1999) suit le parcours des conteneurs qui vont et viennent de Chine, avec, à une extrémité, le chômage à Liverpool et à Los Angeles et, à l'autre, une main-d'œuvre sous-payée en Asie.

22. Cyril Lionel Robert James (1901-1989) est un écrivain, critique littéraire, historien et révolutionnaire caribéen. Grand penseur du panafricanisme, James, lui-même issu d'une colonie, la Trinité-et-Tobago, voit la lutte anticoloniale comme un grand soulèvement international, reliant ensemble les révolutionnaires indépendantistes d'Afrique et les mouvements pour les droits civiques des Afro-Américains. L'une des grandes tensions de son œuvre politique sera de trouver des points de convergence entre l'émancipation des masses prolétaires analysée par la théorie marxiste et l'archipel insurrectionnel contre le colonialisme. Lire à ce sujet Matthieu Renault (2016).

23. Il s'agirait de Jacinto, «le Samson des quais » : un débardeur du début du $\mathrm{XX}^{\mathrm{e}}$ siècle connu pour sa force herculéenne. Pour une analyse approfondie des représentations de Santos dans The Dockers' Museum, voir Day G. (2015).

24. Sur ce point, Sekula semble s'éloigner de Rancière pour qui le "régime esthétique des arts » peut temporairement permettre au spectateur de s'émanciper des dominations qu'il subit dans le monde réel. Cf. Rancière, 2008.

25. https://vimeo.com/129556217

26. Il s'agit d'une paraphrase de Barthes, disant à propos de la publicité, qu'« on n'en finit pas facilement avec la civilisation de la main ».

\section{RÉSUMÉS}

Cet article se propose de revenir sur la pensée et l'œuvre du photographe et théoricien Allan Sekula. Disparu en 2013, Sekula laisse une œuvre dense et engagée. Marqué par Walter Benjamin, il a cherché à relire l'histoire de la photographie dans une perspective matérialiste, où le sens de l'image dépend de l'usage qui en est fait et des discours qui la sous-tendent. Une telle histoire doit montrer les rapports de production et les conflits d'intérêts dans les représentations du travail, comme le montre bien son essai La Photographie, entre travail et capital (1983), centré sur le développement croisé de la photographie et du capitalisme industriel, à partir des images de la mine en particulier. Conscient des forces et des lacunes de son médium, Sekula ne cessera de composer des œuvres jouant sur la fragmentation: fragmentation des corps sous l'effet du management moderne; fragmentation des espaces de transit des marchandises dans un capitalisme maritime et mondialisé ; fragmentation des œuvres elles-mêmes, jouant des liens entre texte et images, pour faire affleurer les rapports de domination, mais aussi les échanges entre l'artiste et son sujet, invisibles et pourtant essentiels à la fabrication de l'image.

Gone in 2013, Allan Sekula is a theorist and a photographer, with a strong and political work. Under the influence of Walter Benjamin, he wanted to write an history of photography in a materialist perspective. He defends the idea that the sense of a picture depends on the discursive and social context, not in the picture itself. Such a history emphasizes relations of production and conflicts of interest in labor's representations. Sekula's essay, Photography, Between Labor and Capital (1983), returns to the evolutions of photography and industrial capitalism, with a focus on the pictures of coal mine. With a keen awareness of strength and weakness of his medium, Sekula used a fragmentary aesthetic: workers' bodies are divided by the modern management; the 
globalisation scattered in strategic and unequal spaces around the world. And by the addition of text in order to reveal a possible ideological domination, or the exchanges between the artist and his subject, his own work shows at last a fragmented reality. Often invisible, these relations are essential in the process of making pictures.

\section{INDEX}

Keywords : Allan Sekula, photography, history of photography, cultural materialism, critical realism

Mots-clés : Allan Sekula, photographie, histoire de la photographie, matérialisme culturel, réalisme critique

\section{AUTEUR}

\section{FLORENT LE DEMAZEL}

Florent Le Demazel a soutenu en 2018 une thèse à l'Université de Lille sur les représentations visuelles du travail ouvrier à différentes époques du capitalisme. Il a participé à la création de la revue Débordements en 2012, à laquelle il collabore depuis. Il y a notamment publié une traduction en français de l'essai La Photographie, entre travail et capital de Allan Sekula. Il réalise également, dans un cadre associatif, des documentaires sur l'histoire du bassin minier du Pas-de-Calais, où il réside. 